\title{
ON EXPLICIT RECIPROCITY LAW OVER FORMAL GROUPS
}

\section{SHAOWEI ZHANG}

Received 16 January 2003

Let $\mathfrak{f}$ be a one-dimensional Lubin-Tate formal group over $\mathbb{Z}_{p}$. Colmez (1998) and Perrin-Riou (1994) proved an explicit reciprocity law for tempered distributions over the formal group $\mathbb{G}_{m}$. In this paper, the general explicit reciprocity law over the formal group $\mathfrak{f}$ is proved.

2000 Mathematics Subject Classification: 11S40.

1. Formal groups. In this section, we review some basic facts on formal groups. De Shalit [3, Chapter I] is the source for this section. We just give the description on Coleman power series and the associated measures, and then study such measures extensively. These measures are the basic examples for our theory. For admissible distributions, see [8].

Let $p$ be an odd prime. For $\alpha \in \mathbb{Z}_{p}^{\times}$, let $\pi=p \alpha$. Let $f_{\pi}(x) \in \mathbb{Z}_{p}[[x]]$ be a Frobenius corresponding to $\pi$, so $f_{\pi}(x) \equiv \pi x(\bmod \operatorname{deg} 2), f_{\pi}(x) \equiv x^{p}(\bmod p)$. Let $\mathfrak{f}$ be the onedimensional Lubin-Tate formal group over $\mathbb{Z}_{p}$ corresponding to $f_{\pi}$, let [+] denote the formal addition. Let $W_{\pi}^{n}:=\left\{x \in \mathbb{C}_{p} \mid f_{\pi}^{(n)}(x)=0\right\}, K_{n}=\mathbb{Q}_{p}\left(W_{\pi}^{n}\right), K_{\infty}=\cup_{n \geq 1} K_{n}$. Hence, $K_{\infty} / \mathbb{Q}_{p}$ is a totally ramified extension with Galois group $\mathbb{Z}_{p}^{\times}$. We call this tower the LubinTate tower corresponding to the formal group $\mathfrak{f}$. Let $R=\mathbb{Z}_{p}[[T]], \mathcal{U}=\varliminf_{n} K_{n}^{\times}$, where the map is with respect to the norm map. Let $\eta: \mathbb{G}_{m} \rightarrow \mathscr{F}_{\pi}$ be an isomorphism, then $\eta \in \widehat{\mathbb{Q}_{p}^{u r}}[[T]]$ and $\eta(T)=\Omega T+\cdots$, such that $\Omega^{\varphi-1}=\alpha$, where $\varphi=$ Frob $_{p}$ is a generator of $\operatorname{Gal}\left(\mathbb{Q}_{p}^{u r} / \mathbb{Q}_{p}\right)$. We have $f \circ \eta=\eta^{\varphi} \circ[p]$, where $[p]=(1+T)^{p}-1$ is the Frobenius of the formal group $\mathbb{G}_{m}$. Let $\omega_{n}=\eta^{\varphi^{-n}}\left(\zeta_{p^{n}}-1\right)$. Then it is easy to see that

(i) $\omega_{n} \in W_{\pi}^{(n)}$,

(ii) $f_{\pi}\left(\omega_{n}\right)=\omega_{n-1}$, as $f_{\pi}\left(\omega_{n}\right)=f_{\pi} \eta^{\varphi^{-n}}\left(\zeta_{p^{n}}-1\right)=\eta^{\varphi^{-n+1}} \circ[p]\left(\zeta_{p^{n}}-1\right)=\eta^{\varphi^{-n+1}}\left(\zeta_{p^{n-1}}-1\right)=\omega_{n-1}$.

Let $T_{\pi}=\lim _{\pi}^{(n)}$ be the Tate module, where the inverse limit is taken with respect to $f_{\pi}$. Let $\kappa: \operatorname{Gal}\left(K_{\infty} / \mathbb{Q}_{p}\right) \rightarrow \mathbb{Z}_{p}^{\times}$be the character given by the action of $G_{\mathbb{Q}_{p}}$ on $T_{\pi}$. If the formal group is $\mathbb{G}_{m}$, this character is just $\chi$, the cyclotomic character. We know that $\kappa=\chi \psi$, where $\psi$ is an unramified character.

Assume $\beta \in \mathcal{U}$, then Coleman's theorem tells us that there is a unique (Coleman) power series $g_{\beta} \in \mathbb{Z}_{p}[[T]]$ such that

(i) $g_{\beta}\left(\omega_{i}\right)=\beta_{i}$, for all $i \geq 1$,

(ii) $g_{\beta}^{\varphi} \circ f_{\pi}(x)=\prod_{w \in W_{\pi}^{1}} g_{\beta}(x[+] w)$.

To get a rough idea for what Coleman power series is, we look at some examples. Consider the $\mathbb{G}_{m}$ case, let $\beta_{n}=\langle 2\rangle+\omega^{-1}(2)\left(\zeta_{p^{n}}-1\right)$, where $\omega$ is the Teichmüller character. Then we have $g_{\beta}(T)=\langle 2\rangle+\omega^{-1}(2) T$. If we take $\beta_{n}=\left(\zeta_{p^{n}}^{a}-1\right) /\left(\zeta_{p^{n}}^{b}-1\right)$, then $g_{\beta}(T)=\left((1+T)^{a}-1\right) /\left((1+T)^{b}-1\right)$. 
Assume $\beta \in \mathcal{U}$ such that $\beta_{n} \equiv 1\left(\bmod \omega_{n}\right)$. Then $g_{\beta}(T) \equiv 1 \bmod (\mathfrak{p}, T)$, where $\mathfrak{p}$ is the maximal ideal in $\mathbb{Z}_{p}$, hence we can define

$$
\widetilde{\log } g_{\beta}(T):=\log g_{\beta}(T)-\frac{1}{p} \sum_{w \in W_{\pi}^{1}} \log g_{\beta}(T[+] w),
$$

property (ii) of the Coleman power series implies that $\widetilde{\log } g_{\beta}(T)$ has integral coefficients. Define an algebraic distribution $\mu_{\beta} \in \mathscr{D}_{\text {alg }}^{+}\left(\mathbb{Z}_{p}, \widehat{\mathbb{Q}_{p}^{u r}}\right)$ such that

$$
\int_{\mathbb{Z}_{p}}(1+T)^{x} \mu_{\beta}(x)=\log g_{\beta} \circ \eta(T) .
$$

Proposition 1.1. (i) The restriction $\left.\mu_{\beta}\right|_{\mathbb{Z}_{p}^{\times}}$is a measure and its Amice transformation is $\widetilde{\log } g_{\beta} \circ \eta(T)$.

(ii) The distribution $\mu_{\beta}$ can be extended to a distribution in $\mathscr{D}_{1}\left(\mathbb{Q}_{p}, \widehat{\mathbb{Q}_{p}^{u r}}\right)^{\Phi=1}$ and has the following Galois property:

$$
\sigma\left(\int_{\mathbb{Q}_{p}} f(x) \mu_{\beta}\right)=\int_{\mathbb{Q}_{p}} f(\psi(\sigma) x) \mu_{\beta}, \quad \forall \sigma
$$

for all $f(x): \mathbb{Q}_{p} \rightarrow \mathbb{Q}_{p}$.

Proof. It is easy to see that

$$
\int_{\mathbb{Z}_{p}^{\times}}(1+T)^{x} \mu_{\beta}=\int_{\mathbb{Z}_{p}}(1+T)^{x} \mu_{\beta}-\int_{p \mathbb{Z} p}(1+T)^{x} \mu_{\beta} .
$$

By property (ii),

$$
g_{\beta} \circ f_{\pi}(X)=\prod_{w \in W_{\pi}^{1}} g_{\beta}(X[+] w) .
$$

Put $X=\eta(T)$, then

$$
g_{\beta} \circ f_{\pi}(\eta(T))=\prod_{\zeta \in \mu_{p}} g_{\beta}(\eta(T)[+] \eta(\zeta-1))=\prod_{\zeta \in \mu_{p}} g_{\beta} \eta(\zeta(1+T)-1) .
$$

By using $f_{\pi} \circ \eta=\eta^{\varphi} \circ[p]$, we see

$$
\left(g_{\beta} \circ \eta\right)^{\varphi} \circ[p]=\prod_{\zeta}\left(g_{\beta} \eta\right)(\zeta(1+T)-1) .
$$

Taking logarithm and using the definition of $\mu_{\beta}$, we have

$$
\varphi\left(\int_{\mathbb{Z} p}(1+[p] T)^{x} \mu_{\beta}\right)=\sum_{\zeta} \int_{\mathbb{Z}_{p}} \zeta^{x}(1+T)^{x} \mu_{\beta}=p \int_{p \mathbb{Z} p}(1+T)^{x} \mu_{\beta} .
$$

We write down this useful property as follows. 
Proposition 1.2. For $\mu_{\beta}$ as above,

$$
\varphi\left(\int_{\mathbb{Z}_{p}}(1+T)^{p x} \mu_{\beta}\right)=p \int_{p \mathbb{Z}_{p}}(1+T)^{x} \mu_{\beta}
$$

Now continue the proof of Proposition 1.1.

The integral

$$
\begin{aligned}
\int_{\mathbb{Z}_{p}^{\times}}(1+T)^{x} \mu_{\beta} & =\log g_{\beta} \eta(T)-\frac{1}{p} \varphi\left(\log g_{\beta} \circ \eta^{\varphi} \circ([p] T)\right) \\
& =\log g_{\beta} \circ \eta-\frac{1}{p} \log g_{\beta} \circ f_{\pi} \circ \eta(T) \\
& =\widetilde{\log } g_{\beta} \circ \eta(T)
\end{aligned}
$$

has integral coefficients, hence $\left.\mu_{\beta}\right|_{\mathbb{Z}_{p}^{\times}}$is a measure. By Proposition 1.2 we extend $\mu_{\beta}$ to $\mathbb{Q}_{p}$ by defining

$$
\int_{p^{-n} \mathbb{Z}_{p}} f(x) \mu_{\beta}=p^{n} \varphi^{-n} \int_{\mathbb{Z}_{p}} f\left(p^{-n} x\right) \mu_{\beta},
$$

for any $f(x)$ with support in $p^{-n} \mathbb{Z}_{p}$. It is easy to see that this definition does not depend on $n$.

To prove the second property, since

$$
\eta(T): \mathbb{G}_{m} \longrightarrow \mathscr{F}_{\pi},
$$

we can show that

$$
\sigma(\eta(T))=\eta\left((1+T)^{\psi(\sigma)}-1\right), \quad \forall \sigma \in G_{\mathbb{Q}_{p}}
$$

To see this, define

$$
h_{\sigma}(T):=\sigma(\eta(T))-\eta\left((1+T)^{\psi(\sigma)}-1\right) .
$$

For $T_{n}=\zeta_{p^{n}}-1, \eta\left(T_{n}\right) \in W_{\pi}^{(n)}$,

$$
\begin{aligned}
(\sigma \eta)\left(\sigma T_{n}\right) & =\sigma\left(\eta\left(T_{n}\right)\right)=[\kappa(\sigma)]_{\pi} \eta\left(T_{n}\right)=\eta\left([\kappa(\sigma)] T_{n}\right) \\
& =\eta\left(\zeta_{p^{n}}^{\kappa(\sigma)}-1\right)=\eta\left(\left(1+\sigma T_{n}\right)^{\psi(\sigma)}-1\right),
\end{aligned}
$$

hence $h_{\sigma}\left(\sigma T_{n}\right)=0$ for all $n \geq 1$. The function $h_{\sigma}(T)$ has infinitely many zeros by Weierstrass lemma, then $h_{\sigma}(T)=0$.

From this property, we see that

$$
\begin{aligned}
\sigma\left(\int_{\mathbb{Z}_{p}}(1+T)^{x} \mu_{\beta}\right) & =\sigma\left(\log g_{\beta} \circ \eta(T)\right)=\log \mathcal{g}_{\beta} \circ \sigma(\eta(T)) \\
& =\log g_{\beta} \circ \eta\left((1+T)^{\psi(\sigma)}-1\right)=\int_{\mathbb{Z}_{p}}(1+T)^{\psi(\sigma) x} \mu_{\beta},
\end{aligned}
$$


so for general $f$, we have

$$
\sigma\left(\int_{\mathbb{Z} p} f(x) \mu_{\beta}\right)=\int_{\mathbb{Z}_{p}} f(\psi(\sigma) x) \mu_{\beta}
$$

From the extension definition (1.11) of $\mu_{\beta}$, we have, for all $f$,

$$
\sigma\left(\int_{\mathbb{Q}_{p}} f(x) \mu_{\beta}\right)=\int_{\mathbb{Q}_{p}} f(\psi(\sigma) x) \mu_{\beta}
$$

To show that $\mu_{\beta}$ is 1-admissible, by definition and [8, Proposition 3.3], we only need to show that $p^{n(1-j)} \int_{a+p^{n} \mathbb{Z}_{p}}(x-a)^{j} \mu_{\beta}$ is $r$-bounded for $j=0,1$. For $j=0$, if $a \neq 0$, then since $\left.\mu_{\beta}\right|_{\mathbb{Z}_{p}^{\times}}$is a measure, the integral $p^{n} \int_{a+p^{n} \mathbb{Z}_{p}} \mu_{\beta}$ is always bounded. If $a=0$, then $p^{n} \int_{p^{n} \mathbb{Z}_{p}} \mu_{\beta}=\varphi^{n}\left(\int_{\mathbb{Z}_{p}} \mu_{\beta}\right)=\varphi^{n} \log g_{\beta}(0)=\log \mathcal{g}_{\beta}(0)$, hence bounded.

For $j=1$, if $a \neq 0$, then $\int_{a+p^{n} \mathbb{Z}_{p}} x \mu_{\beta}$ is bounded. If $a=0$, then $\int_{p^{n} \mathbb{Z}_{p}} x \mu_{\beta}=\varphi^{n}\left(\int_{\mathbb{Z}_{p}} x \mu_{\beta}\right)=$ $\varphi^{n}\left(\Omega \cdot\left(g_{\beta}^{\prime}(0) / g_{\beta}(0)\right)\right)=\alpha^{n} \Omega\left(g_{\beta}^{\prime}(0) / g_{\beta}(0)\right)$, hence bounded.

Next, we will restrict to the $\mathbb{G}_{m}$ case. $\mu_{\beta}$ is an example which is a distribution on $\mathbb{Q}_{p}$ but not a measure. We will show an example of distribution which is not a tempered distribution. $\mu_{\beta}$ can be extended to negative power. For $k>0$, define

$$
\int_{\mathbb{Z} p} x^{-k} \mu_{\beta}=\left(1-p^{-k-1}\right)^{-1} \int_{\mathbb{Z}_{p}^{\times}} x^{-k} \mu_{\beta}
$$

define $v_{\beta} \in \mathscr{D}_{\text {alg }}^{+}\left(\mathbb{Z}_{p}, K_{\infty, \text { cyc }}\right)$ such that

$$
\int_{a+p^{n} \mathbb{Z}_{p}} x^{k} v_{\beta}:=p^{n k} k ! \int_{\mathbb{Z}_{p}} \varepsilon\left(\frac{a x}{p^{n}}\right) \frac{\mu_{\beta}}{x^{k}}, \quad k \geq 0
$$

The relation between $\mu_{\beta}$ and $v_{\beta}$ is that

$$
v_{\beta} \otimes \frac{e}{t}=\mathscr{F}_{\mathrm{alg}}\left(\mu_{\beta} \otimes \frac{e}{t}\right) .
$$

LEMMA 1.3. The distribution $v_{\beta}$ is a distribution over $\mathbb{Z}_{p}$, but it is not a tempered distribution if $\widetilde{\log } g_{\beta}(T) \not \equiv \widetilde{\log } g_{\beta}(0)\left(\bmod \left(p, T^{p-1}\right)\right)$.

Proof. The additivity of $v_{\beta}$ follows from the relation

$$
\sum_{i=0}^{p-1} \varepsilon\left(\frac{\left(a+i p^{n}\right) x}{p^{n+1}}\right)=\left\{\begin{array}{ll}
p \varepsilon\left(\frac{a x}{p^{n+1}}\right), & x \equiv 0 \\
0, & x \neq 0
\end{array}(\bmod p) .\right.
$$

Assume $\widetilde{\log } g_{\beta}(T)=\sum a_{i} T^{i}$, since $\widetilde{\log } g_{\beta} \not \equiv 0 \bmod \left(p, T^{p-1}\right)$, hence there exists $i, 1 \leq i \leq$ $p-1$, such that $a_{i} \not \equiv 0(\bmod p)$. Then $\int_{1+p^{n} \mathbb{Z}_{p}} v_{\beta}=\log \beta_{n}$ has denominator with valuation $n-1-i /(p-1)$, so $v_{\beta}$ is not a measure. 
If $v_{\beta}$ is $r$-admissible, assume $r \in \mathbb{N}$, then for all $X$,

$$
\sup _{a \in X}\left\|p^{[n(r-j)]} \int_{a+p^{n} \mathbb{Z}_{p}}(x-a)^{j} v_{\beta}\right\|
$$

is $r$-bounded. Taking $j=r$, we would have that $\int_{a+p^{n} \mathbb{Z}_{p}}(x-a)^{r} v_{\beta}$ is bounded when $n \rightarrow \infty$.

We will calculate the valuation of $\int_{a+p^{n} \mathbb{Z}_{p}}(x-a)^{r} v_{\beta}$. For $a=1$,

$$
\int_{a+p^{n} \mathbb{Z}_{p}}(x-1)^{r} v_{\beta}=\sum_{k=0}^{r}\left(\begin{array}{l}
r \\
k
\end{array}\right) \cdot(-1)^{r-k} \int_{1+p^{n} \mathbb{Z}_{p}} x^{k} v_{\beta}=\sum_{k=0}^{r} d_{k} .
$$

Claim 1.4. $v\left(d_{k}\right)>i /(p-1)-(n-1)=v\left(d_{0}\right)$.

To prove the claim, keeping using the relation

$$
\int_{p \mathbb{Z}_{p}} \varepsilon\left(\frac{x}{p^{m}}\right) \frac{\mu_{\beta}}{x^{k}}=\frac{1}{p^{k+1}} \int_{\mathbb{Z}_{p}} \varepsilon\left(\frac{x}{p^{m-1}}\right) \frac{\mu_{\beta}}{x^{k}}
$$

and the decomposition

$$
\mathbb{Z}_{p}=\mathbb{Z}_{p}^{\times} \cup p \mathbb{Z}_{p}^{\times} \cup \cdots \cup p^{n-1} \mathbb{Z}_{p}^{\times} \cup p^{n} \mathbb{Z}_{p}
$$

we have

$$
\begin{aligned}
& \int_{1+p^{n} \mathbb{Z}_{p}} x^{k} v_{\beta} \\
& =p^{k n} \cdot k ! \int_{\mathbb{Z}_{p}} \varepsilon\left(\frac{x}{p^{n}}\right) \frac{\mu_{\beta}}{x^{k}} \\
& =p^{k n} \cdot k !\left(\sum_{i=0}^{n-1} \int_{p^{i} \mathbb{Z}_{p}^{\times}} \varepsilon\left(\frac{x}{p^{n}}\right) \frac{\mu_{\beta}}{x^{k}}+\int_{p^{n} \mathbb{Z}_{p}} \varepsilon\left(\frac{x}{p^{n}}\right) \frac{\mu_{\beta}}{x^{k}}\right) \\
& =p^{k n} \cdot k !\left(\sum_{i=0}^{n-1} \frac{1}{p^{i(k+1)}} \int_{\mathbb{Z}_{p}^{\times}} \varepsilon\left(\frac{x}{p^{n-i}}\right) \frac{\mu_{\beta}}{x^{k}}+\frac{1}{p^{n(k+1)}} \int_{\mathbb{Z}_{p}} \frac{\mu_{\beta}}{x^{k}}\right) \\
& =p^{k n} \cdot k !\left(\sum_{i=0}^{n-1} \frac{1}{p^{i(k+1)}} \int_{\mathbb{Z}_{p}^{\times}} \varepsilon\left(\frac{x}{p^{n-i}}\right) \frac{\mu_{\beta}}{x^{k}}+\frac{1}{p^{n(k+1)}}\left(1-p^{-k-1}\right)^{-1} \int_{\mathbb{Z}_{p}^{\times}} \frac{\mu_{\beta}}{x^{k}}\right) .
\end{aligned}
$$

The last two terms for $d_{0}$ equal

$$
\frac{1}{p^{n-1}} \int_{\mathbb{Z}_{p}^{\times}} \varepsilon\left(\frac{x}{p}\right) \mu_{\beta}+\frac{p-1}{p^{n-1}} \int_{\mathbb{Z}_{p}^{\times}} \mu_{\beta}=\frac{1}{p^{n-1}}\left(\widetilde{\log } g_{\beta}\left(\zeta_{1}-1\right)+(p-1) \widetilde{\log } g_{\beta}(0)\right)
$$

Assume $\widetilde{\log } g_{\beta}(T)=a_{0}+a_{1} T+\cdots$. From the hypothesis we know that there exists a minimal $i$ with $1 \leq i<p-1$ such that $a_{i} \neq \equiv 0 \bmod p$, hence the above expression equals

$$
\frac{1}{p^{n-1}}\left(p a_{0}+a_{1}\left(\zeta_{1}-1\right)+\cdots+a_{i}\left(\zeta_{1}-1\right)^{i}+\cdots\right),
$$


and therefore $v\left(d_{0}\right)=i /(p-1)-(n-1)$. For $k>0$, we easily see that $v\left(d_{k}\right) \geq k-$ $(n-1)>v\left(d_{0}\right)$, hence the claim follows. The sequence $\int_{1+p^{n} \mathbb{Z}_{p}}(x-1)^{r} v_{\beta}$ could not be bounded, hence this completes the proof of the lemma.

If $r \notin \mathbb{R}$, taking $j=[r]$, then $p^{[n(r-[r])]} \int_{1+p^{n} \mathbb{Z}_{p}}(x-1)^{[r]} v_{\beta}$ cannot tend to zero.

2. Galois action on $B_{d R}$. Let $\mathfrak{f}$ be a one-dimensional height-one formal group over $\mathbb{Z}_{p}$, let $\pi=p \alpha$ be the uniformizer with $\alpha \in \mathbb{Z}_{p}^{\times}$, and let $f$ be a Frobenius power series corresponding to $\mathfrak{f}$, then we have $f(x) \equiv \pi x(\bmod \operatorname{deg} 2), f(x) \equiv x^{p}(\bmod p)$. Let $\eta(x)$ : $\mathbb{G}_{m} \rightarrow \mathfrak{f}$ which is an isomorphism, such that $\eta(x)=\Omega x+\cdots$, where $\Omega$ is a $p$-adic unit with $\Omega^{\varphi-1}=\alpha$. Let $\omega_{n}=\eta^{\varphi^{-n}}\left(\zeta_{p^{n}}-1\right), K_{n}=\mathbb{Q}_{p}\left(\omega_{n}\right)$, and $K_{\infty}=\cup_{n \geq 0} K_{n}$, let $T_{\pi}$ be the Tate module, and let $\kappa: \operatorname{Gal}\left(K_{\infty} / \mathbb{Q}_{p}\right) \rightarrow \mathbb{Z}_{p}^{\times}$be the character given by the action on $T_{\pi}$, then we know that $\kappa=\chi \psi$ with $\psi$ an unramified character such that $\psi\left(\right.$ Frob $\left._{p}\right)=\alpha$ and $\sigma\left(\omega_{n}\right)=[\kappa(\sigma)] \omega_{n}$, where for $a \in \mathbb{Z}_{p},[a]$ denotes the unique endomorphism of $\mathfrak{f}$ such that $[a]=a X+\cdots$ (see [4, Section 20.1]). Let $\Xi$ be the completion of $\mathbb{Q}_{p}^{u r}$. Let $t_{\pi}=\Omega t$. Then $t_{\pi}$ has the property that $\sigma\left(t_{\pi}\right)=\kappa(\sigma) t_{\pi}, \varphi\left(t_{\pi}\right)=\pi t_{\pi}$.

If $x \in K_{\infty}$ and $n \in \mathbb{N}$, we define $T_{n}(x):=\left(1 / p^{m}\right) \operatorname{Tr}_{K_{m} / K_{n}}(x)$ for $m \gg 1$. We extend $T_{n}$ to a map from $K_{\infty}\left(\left(t_{\pi}\right)\right)$ to $K_{n}\left(\left(t_{\pi}\right)\right)$ by $T_{n}\left(\sum a_{k} t_{\pi}^{k}\right)=\sum T_{n}\left(a_{k}\right) t_{\pi}^{k}$. Note that this definition does not depend on the choice of $t_{\pi}$ since the different choice only differs a multiple in $\mathbb{Q}_{p}$ and $T_{n}$ is $\mathbb{Q}_{p}$-linear. We also define $\operatorname{Tr}_{/ K_{n}}=\left(1 /\left[K_{m}: K_{n}\right]\right) \operatorname{Tr}_{K_{m} / K_{n}}(x)$ for $m \gg 1$.

Recall that $\mathscr{R}$ is the projective limit of the following diagram:

$$
\bar{O} \leftarrow \bar{O} \leftarrow \cdots
$$

For $x \in \mathscr{R}, x=\left(x_{n}\right)_{n \in \mathbb{N}}, x_{n+1}^{p}=x_{n}$. Choose $\tilde{x}_{n} \in O_{\mathbb{C}_{p}}$ such that $\tilde{x}_{n} \equiv x_{n}(\bmod p)$.

LEMMA 2.1. The limit $\lim _{n \rightarrow \infty} f^{(m)}\left(\tilde{x}_{n+m}\right)$ exists and does not depend on the choice of $\tilde{x}_{n}$. Denote it by $x^{(m)}$ and then we have $f\left(x^{(m)}\right)=x^{(m-1)}$.

Proof. We first prove that $f$ has the property, for $y \in \mathscr{R}$,

$$
f^{(n)}(x+p y) \equiv f^{(n)}(x)\left(\bmod p^{n+1} y\right) .
$$

This is true for $n=1$ from the definition of $f$. Assume it is true for $n$, then we have $f^{(n)}(x+p y)=f^{(n)}(x)+p^{n+1} y z$ for some $z \in \mathscr{R}$, hence

$$
\begin{aligned}
f^{(n+1)}(x+p y) & =f\left(\left(f^{(n)}(x)+p^{n+1} y z\right)\right) \\
& =f^{(n+1)}(x)\left(\bmod p \cdot p^{n+1} y z\right) \\
& =f^{(n+1)}(x)\left(\bmod p^{n+2} y\right) .
\end{aligned}
$$

From this we see that

$$
\begin{aligned}
f^{(n)}\left(\tilde{x}_{n+m}\right)-f^{(n+1)}\left(\tilde{x}_{n+m+1}\right) & =f^{(n)}\left(\tilde{x}_{n+m}\right)-f^{(n+1)}\left(\tilde{x}_{n+m}+p y\right) \\
& \equiv 0\left(\bmod p^{n+1}\right),
\end{aligned}
$$

hence the lemma follows. 
On the other hand, if $\left(x^{(m)}\right)$ satisfies the relation $f\left(x^{(m)}\right)=x^{(m-1)}$, then $\left(\bar{x}^{(m)}\right) \in \mathscr{R}$, hence $\mathscr{R}$ is one-to-one corresponding to

$$
\left\{\left(x^{(m)}\right)_{m \in \mathbb{N}} \mid f\left(x^{(m)}\right)=x^{(m-1)}\right\} .
$$

Let $W=\left\{w \in \mathbb{C}_{p} \mid \exists n\right.$, s.t. $\left.[\pi]^{n} w=0\right\}$. For $w \in W$, if $n \in \mathbb{N}$ such that $[\pi]^{n} w \neq 0$, $[\pi]^{n+1} w=0$, then we say that $w$ has order $n$. To $w \in W$, we can associate an element $\bar{w}=\left(w, f^{(-1)}(w), f^{(-2)}(w), \ldots\right)$. The association is not unique.

LEMMA 2.2. All elements of $\mathbb{C}_{p}^{G_{K \infty}}$ can be written in the form $x=\sum_{w \in W} a_{w}(x) w$ with $a_{w}(x) \in \mathbb{Q}_{p}$ tending to zero when the order of $w$ tends to infinity.

Proof. Since $K_{\infty}=\overline{\mathbb{Q}}_{p}^{G_{K \infty}}, W$ is a base of $O_{K_{\infty}} / \mathbb{Z}_{p}$ and $\mathbb{C}_{p}^{G_{K \infty}}$ is a separated completion of $K_{\infty}$ with respect to $p$-adic topology, hence the proof follows from Tate-Sen-Ax theorem.

Proposition 2.3. (i) The field $K_{\infty}\left(\left(t_{\pi}\right)\right)$ is dense in $\left(B_{d R}^{+}\right)^{G_{K_{\infty}}}$ and $T_{n}$ can be extended to a continuous $\mathbb{Q}_{p}$-linear map from $\left(B_{d R}^{+}\right)^{G_{K \infty}}$ to $K_{n}\left(\left(t_{\pi}\right)\right)$.

(ii) If $F \in\left(B_{d R}^{+}\right)^{G_{K_{\infty}}}$, then $\lim _{n \rightarrow \infty} p^{n} T_{n}(F)=F$.

Proof. (i) For all $x \in\left(B_{d R}^{+}\right)^{G_{K \infty}}, \theta(x) \in \mathbb{C}_{p}^{G_{K \infty}}$, hence $\theta(x)=\sum_{w \in W} a_{w}(x) w$ for some $a_{w}(x) \in \mathbb{Q}_{p}$ from Lemma 2.2. Let

$$
R(x)=t_{\pi}^{-1}\left(x-\sum_{w \in W} a_{w}(x)[\bar{w}]\right) \in\left(B_{d R}^{+}\right)^{G_{K \infty}},
$$

so we can repeat the above process and get

$$
x=t_{\pi}^{k+1} R^{k+1}(x)-\sum_{i=0}^{k} t_{\pi}^{i}\left(\sum_{w} a_{w}\left(R^{(i)}(x)\right)[\bar{w}]\right),
$$

and this shows that $K_{\infty}\left(\left(t_{\pi}\right)\right)$ is dense in $\left(B_{d R}^{+}\right)^{G_{K \infty}}$.

(ii) For $F \in K_{\infty}, F \in K_{m_{0}}$ for some $m_{0}$, hence for $n \geq m_{0}, T_{n}(F)=\left(1 / p^{m}\right) \operatorname{Tr}_{K_{m} / K_{n}}(F)=$ $\left(1 / p^{n}\right) F$. Hence, $\lim _{n \rightarrow+\infty} p^{n} T_{n}(F)=F$. By the definition of $T_{n}$ on $K_{\infty}\left(\left(t_{\pi}\right)\right)$ we have for $F \in K_{\infty}\left(\left(t_{\pi}\right)\right), \lim _{n \rightarrow+\infty} p^{n} T_{n}(F)=F$.

For $F \in\left(B_{d R}^{+}\right)^{G_{K \infty}}$, (i) shows that we can take $F_{k} \in K_{\infty}\left(\left(t_{\pi}\right)\right)$ such that $\lim _{k \rightarrow+\infty} F_{k}=F$. Hence

$$
\lim _{n \rightarrow+\infty} p^{n} T_{n}(F)=\lim _{n \rightarrow+\infty} p^{n} T_{n}\left(\lim _{k \rightarrow+\infty} F_{k}\right)=\lim _{k \rightarrow+\infty} \lim _{n \rightarrow+\infty} p^{n} T_{n}\left(F_{k}\right)=\lim _{k \rightarrow+\infty} F_{k}=F .
$$

We can change the order of the limit since $p^{n} T_{n}$ is continuous.

Recall that LA denotes the space of locally analytic functions with compact support in $\mathbb{Q}_{p}$ taking values in $\mathbb{Q}_{p}$. For a $p$-adic Banach space $A$, define $\mathscr{D}_{\text {cont }}\left(\mathbb{Q}_{p}, A\right)=$ $\operatorname{Hom}_{\text {cont }}(\mathrm{LA}, A)$ with respect to Morita topology. Define

$$
\mathscr{D}_{\text {cont }}\left(\mathbb{Q}_{p}, \Xi\right)^{\psi}:=\left\{\mu \in \mathscr{D}_{\text {cont }}\left(\mathbb{Q}_{p}, \Xi\right) \mid \sigma\left(\int_{\mathbb{Q}_{p}} f(x) \mu\right)=\int_{\mathbb{Q}_{p}} f(\psi(\sigma) x) \mu, \forall \sigma \in G_{\mathbb{Q}_{p}}\right\} .
$$


For $\mu \in \mathscr{D}_{\text {cont }}\left(\mathbb{Q}_{p}, \Xi\right)^{\psi}$ with compact support, we can define an element in $B_{d R}$ as

$$
\mathscr{F}_{\text {cont }}(\mu)=\int_{\mathbb{Q}_{p}}\left[\varepsilon^{x}\right] \mu .
$$

Colmez called this element as the continuous Fourier transformation of $\mu$ and we continue using his notation. Recall from $\left[8\right.$, Section 4] that $\mu \in \mathscr{D}_{\text {cont }}\left(\mathbb{Q}_{p}, \Xi\right)$ is said to be of order $r \in \overline{\mathbb{R}}^{+}$if for all open compact set $X \subset \mathbb{Q}_{p}$ and all $j \geq 0$, the following sequence is $r$-bounded:

$$
\sup _{a \in X}\left\|p^{E(n(r-j))} \int_{a+p^{n} \mathbb{Z}_{p}}(x-a)^{j} \mu\right\| .
$$

Define $\mathbf{B}_{\text {cont }}^{+}:=\cap_{n \geq 0} \varphi^{n}\left(\mathbf{B}_{\text {max }}^{+}\right)$. An element $F \in \mathbf{B}_{\text {cont }}^{+}$is said to be of order $r$ if the sequence $p^{[n r]} \varphi^{-n} F$ is $r$-bounded. The power series $A(T)=\sum a_{n} T^{n} \in \Xi[[T]]$ is said to be of order $r$ if $n^{-r}\left|a_{n}\right|$ is $r$-bounded. Define $\Xi[[T]]^{\psi}:=\{h(T) \in \Xi[[T]]$ such that for all $\left.\sigma \in G_{\mathbb{Q}_{p}}, \sigma(h(T))=h\left((1+T)^{\psi(\sigma)}-1\right)\right\}$.

Proposition 2.4. (i) For $\mu \in \mathscr{D}_{\text {cont }}\left(\mathbb{Q}_{p}, \Xi\right)^{\psi}$, $\mathscr{F}_{\text {cont }}(\mu) \in\left(B_{\text {cont }}^{+}\right)^{G_{K \infty}}$.

(ii) For $\mu \in \mathscr{D}_{\text {cont }}\left(\mathbb{Z}_{p}, \Xi\right)^{\psi}$, the Amice transformation $\mathscr{A}_{\mu}(T)$ is in $\Xi[[T]]^{\psi}, \mu$ has order $r$ if and only if $\mathscr{A}_{\mu}$ has.

(iii) For $\mu \in \mathscr{D}_{\text {cont }}\left(\mathbb{Z}_{p}, \Xi\right)^{\psi}$, if $\mu$ has order $r$, then $\mathscr{F}_{\text {cont }}(\mu)$ has order $r$.

(iv) For a crystalline representation $V$, for $\mu \in \mathscr{D}_{\text {cont }}\left(\mathbb{Z}_{p}, \Xi \otimes D(V)\right)^{\psi}$, if $\mu$ has order $r$, then $\mathscr{F}_{\text {cont }}(\mu)$ has order $r+r(V)$, where $r(V)=\min \left\{k \in \mathbb{Z} \mid \varphi(d)=p^{k} d\right.$ for some $d \in D(V)\}$.

Proof. (i) For $\sigma \in G_{\mathbb{Q}_{p}}$ and $\mu \in \mathscr{D}_{\text {cont }}\left(\mathbb{Q}_{p}, \Xi\right)^{\psi}$,

$$
\sigma\left(\mathscr{F}_{\text {cont }}(\mu)\right)=\sigma\left(\int_{\mathbb{Q}_{p}}\left[\varepsilon^{x}\right] \mu\right)=\int_{\mathbb{Q}_{p}}\left[\varepsilon^{\psi(\sigma) x x(\sigma)}\right] \mu=\int_{\mathbb{Q}_{p}}\left[\varepsilon^{\kappa(\sigma) x}\right] \mu .
$$

If $\kappa(\sigma)=1$, then $\sigma\left(\mathscr{F}_{\text {cont }}(\mu)\right)=\mathscr{F}_{\text {cont }}(\mu)$, so $\mathscr{F}_{\text {cont }}(\mu) \in\left(B_{\text {max }}^{+}\right)^{G_{K_{\infty}}}$. On the other hand, let $F_{n}=\int_{\mathbb{Q}_{p}}\left[\varepsilon_{n}^{\alpha^{-n} x}\right] \mu$; we have

$$
\varphi\left(F_{n}\right)=\varphi^{n}\left(\int_{\mathbb{Q}_{p}}\left[\varepsilon_{n}^{\alpha^{-n} x}\right] \mu\right)=\int_{\mathbb{Q}_{p}}\left[\varepsilon^{x}\right] \mu
$$

and this shows that $\mathscr{F}_{\text {cont }}(\mu) \in\left(\mathbf{B}_{\text {cont }}^{+}\right)^{G_{K_{\infty}}}$.

(ii) The Galois action gives

$$
\sigma\left(\mathscr{A}_{\mu}(T)\right)=\sigma \int_{\mathbb{Z}_{p}}(1+T)^{x} \mu=\int_{\mathbb{Z}_{p}}(1+T)^{\psi(\sigma) x} \mu=\mathscr{A}_{\mu}\left((1+T)^{\psi(\sigma)}-1\right),
$$

hence $\mathscr{A}_{\mu}(T) \in \Xi[[T]]^{\psi}$.

The second statement can be found in [1].

(iii) Assume $\mu$ has order $r$, hence $\mathscr{A}_{\mu}(T)$ has order $r$ by (ii).

CASE 1. Assume $r \in \mathbb{N}$, then there exists a constant $C>0$ such that $\left|a_{k}\right| \leq C k^{r}$, that is, $-v_{p}\left(a_{k}\right) \leq(\log C+r \log k) / \log p$ (here the $\log$ is the logarithmic function on real variable). The real function $f(x, a, r)=a x-r(\log x / \log p)$ has a minimum $g(a, r)$ at 
$x=r / a \log p$. For $x \in \mathbb{R}_{>0}$, we have

$$
\begin{aligned}
v_{p}\left(a_{k}\right)+r n+\frac{k}{(p-1) p^{n-1}} & \geq r n+\frac{k}{(p-1) p^{n-1}}-r \frac{\log k}{\log p}-\frac{\log C}{\log p} \\
& =f\left(\frac{k}{p^{n}}, \frac{p}{p-1}, r\right)-\frac{\log C}{\log p} \\
& \geq g\left(\frac{p}{p-1}, r\right)-\frac{\log C}{\log p} .
\end{aligned}
$$

Take $m \leq-1+g(p /(p-1), r)-\log C / \log p$ and recall that

$$
\begin{gathered}
F_{n}=\int_{\mathbb{Q}_{p}}\left[\varepsilon_{n}^{\alpha^{-n} x}\right] \mu=\sum a_{k}\left(\left[\varepsilon_{n}^{\alpha^{-n}}\right]-1\right)^{k}, \\
\left\|F_{n}\right\|=\sup _{k} p^{-\left(v_{p}\left(a_{k}\right)+k v_{p}\left(\left[\varepsilon_{n}^{\alpha^{-n}}\right]-1\right)\right)}=\sup _{k} p^{-\left(v_{p}\left(a_{k}\right)+k /(p-1) p^{n-1}\right)} ;
\end{gathered}
$$

we get

$$
\left\|p^{n r} F_{n}\right\|=\sup _{k} p^{-\left(n r+v_{p}\left(a_{k}\right)+k /(p-1) p^{n-1}\right)} \leq p^{-m}
$$

hence $\mathscr{F}_{\text {cont }}(\mu)$ is $r$-bounded.

CASE 2. For $r \in \overline{\mathbb{R}}^{+} \backslash \mathbb{R}^{+}$, then we have $\lim _{n \rightarrow+\infty} n^{-r}\left|a_{n}\right|=0$. Hence, we can choose a sequence $c_{k} \rightarrow 0$ such that $\left|a_{k}\right|<c_{k} k^{r}$, then the above proof shows that we can take

$$
m_{k}=\left[-1+g\left(\frac{p}{p-1}, r\right)-\frac{\log c_{k}}{\log p}\right]
$$

then $\left\|p^{[n r]} F_{n}\right\| \leq p^{-m_{n}}$ hence tends to zero.

(iv) Assume $d_{1}, \ldots, d_{k}$ are a base of $D(V)$ and $\mu \in \mathscr{D}\left(\mathbb{Q}_{p}, \Xi \otimes D(V)\right)^{\psi}$, then $\mathscr{F}_{\text {cont }}(\mu)=$ $\sum b_{i} \otimes d_{i}$ for some $b_{i} \in \mathbf{B}_{\text {cont }}^{+}$with order $r, p^{-n r} \mid \varphi^{-n}\left(b_{i}\right) \| \leq c_{n}$ for some $c_{n}\left(c_{n}\right.$ is bounded if $r \in \mathbb{R}$, otherwise $\left.c_{n} \rightarrow 0\right)$, so $p^{-n(r+r(V))}\left|\varphi^{-n}\left(b_{i} \otimes d_{i}\right)\right| \leq c_{n}\left|d_{i}\right|$, hence $\mathscr{F}_{\text {cont }}(\mu)$ is $(r+r(v))$-bounded.

REMARK 2.5. Properties (iii) and (iv) are even true for $\mu$ has support in $\mathbb{Q}_{p}$. To prove this we only need to extend (ii) to this case.

LEMMA 2.6. (i) For $m \geq n \geq 1$,

$$
\sum_{\sigma \in \operatorname{Gal}\left(K_{m} / K_{n}\right)} \varepsilon(\kappa(\sigma) x)= \begin{cases}p^{m-n} \varepsilon(x), & \text { if } x \in p^{-n} \mathbb{Z}_{p}, \\ 0, & \text { otherwise. }\end{cases}
$$

(ii) For $m>0$,

$$
\sum_{\sigma \in \operatorname{Gal}\left(K_{m} / K_{0}\right)} \varepsilon(\kappa(\sigma) x)=p^{m} 1_{\mathbb{Z}_{p}}-p^{m-1} 1_{p^{-1} \mathbb{Z}_{p}}
$$


Proof. (i) If $x \in p^{-n} \mathbb{Z}_{p}$, then

$$
\sum_{\sigma \in G\left(K_{m} / K_{n}\right)} \varepsilon(\kappa(\sigma) x)=\sum_{a=0}^{p^{m-n}-1} \varepsilon\left(\left(1+a \cdot p^{n}\right) x\right)=p^{m-n} \varepsilon(x) .
$$

Otherwise,

$$
\sum_{\sigma \in G\left(K_{m} / K_{n}\right)} \varepsilon(\kappa(\sigma) x)=\varepsilon(x) \sum_{a=0}^{p^{m-n}-1} \varepsilon\left(\frac{a b}{p^{k}}\right)=0
$$

with some $b \neq 0$ a $p$-unit, $k \geq 1$.

(ii) If $x \in \mathbb{Z}_{p}$, then

$$
\sum_{\sigma \in G\left(K_{m} / K_{0}\right)} \varepsilon(\kappa(\sigma) x)=p^{m}-p^{m-1}
$$

If $x \in p^{-1} \mathbb{Z}_{p} \backslash \mathbb{Z}_{p}$, then

$$
\sum_{\sigma \in G\left(K_{m} / K_{0}\right)} \varepsilon(\kappa(\sigma) x)=p^{m-1} \sum_{a=1}^{p-1} \varepsilon\left(\frac{a}{p}\right)=p^{m-1} \cdot(-1)=-p^{m-1} .
$$

And it is easy to see that if $x \notin p^{-1} \mathbb{Z}_{p}$, then

$$
\sum_{\sigma \in G\left(K_{m} / K_{0}\right)} \varepsilon(\kappa(\sigma) x)=0
$$

So the sum equals $1_{\mathbb{Z}_{p}} \cdot\left(p^{m}-p^{m-1}\right)-1_{p^{-1} \mathbb{Z}_{p} \backslash \mathbb{Z}_{p}} p^{m-1}=p^{m} 1_{\mathbb{Z}_{p}}-p^{m-1} 1_{p^{-1} \mathbb{Z}_{p}}$.

Proposition 2.7. If $\mu \in \mathscr{D}_{\text {cont }}\left(\mathbb{Q}_{p}, \Xi\right)^{\psi}$, then

$$
\begin{aligned}
& T_{n}\left(\mathscr{F}_{\operatorname{cont}}(\mu)\right)=\sum_{k=0}^{+\infty} t^{k}\left(p^{-n} \int_{p^{-n} \mathbb{Z}_{p}} \varepsilon(x) \frac{x^{k}}{k !} \mu\right), \quad \text { if } n \geq 1, \\
& T_{0}\left(\mathscr{F}_{\operatorname{cont}}(\mu)\right)=\sum_{k=0}^{+\infty} t^{k}\left(\int_{\mathbb{Z}_{p}} \frac{x^{k}}{k !} \mu-p^{-1} \int_{p^{-1} \mathbb{Z}_{p}} \frac{x^{k}}{k !} \mu\right) .
\end{aligned}
$$

Proof. The Fourier transformation gives

$$
\begin{aligned}
\mathscr{F}_{\text {cont }}(\mu) & =\int_{\mathbb{Q}_{p}}\left[\varepsilon^{x}\right] \mu=\int_{\mathbb{Q}_{p}} \varepsilon(x) \exp (t x) \mu \\
& =\int_{\mathbb{Q}_{p}} \varepsilon(x) \sum_{k=0}^{\infty} \frac{(t x)^{k}}{k !} \mu=\sum_{k=0}^{\infty} t_{\pi}^{k} \int_{\mathbb{Q}_{p}} \varepsilon(x) \frac{x^{k}}{\Omega^{k} \cdot k !} \mu .
\end{aligned}
$$


To prove the first identity, we need to show that

$$
T_{n}\left(\int_{\mathbb{Q}_{p}} \varepsilon(x) \frac{x^{k}}{\Omega^{k} \cdot k !} \mu\right)=p^{-n} \int_{p^{-n} \mathbb{Z}_{p}} \varepsilon(x) \frac{x^{k}}{\Omega^{k} \cdot k !} \mu
$$

(note that the right-hand side is indeed in $K_{n}$ ).

Now, assume $\mu$ has compact support $p^{-m} \mathbb{Z}_{p}$ for some $m$, then

$$
\begin{aligned}
& T_{n}\left(\int_{p^{-m_{\mathbb{Z}_{p}}}} \varepsilon(x) \frac{x^{k}}{\Omega^{k}} \mu\right)=\frac{1}{p^{m}} \sum_{\sigma \in G\left(K_{m} / K_{n}\right)} \sigma\left(\int_{p^{-m_{\mathbb{Z}_{p}}}} \varepsilon(x) \frac{x^{k}}{\Omega^{k}} \mu\right) \\
& =\frac{1}{p^{m}} \sum_{\sigma} \int_{p^{-m} \mathbb{Z}_{p}} \varepsilon(\kappa(\sigma) x) \frac{x^{k}}{\Omega^{k}} \mu \\
& =p^{-n} \int_{p^{-n} \mathbb{Z}_{p}} \varepsilon(x) \frac{x^{k}}{\Omega^{k}} \mu .
\end{aligned}
$$

The same proof works for the second formula.

The Galois action on $B_{d R}$ has the following property.

LEMMA 2.8. Suppose $V$ is a $p$-adic representation of $G_{K_{\infty}}$, then $\left(B_{d R} \otimes V\right)^{G_{K_{\infty}}}$ is a finite-dimensional vector space over $B_{d R}^{G_{K_{\infty}}}$ with dimension $\operatorname{dim}_{\mathbb{Q}_{p}} V$ and can have a base consisting of elements in $\left(B_{\max }^{\varphi=1} \otimes V\right)^{G_{K_{\infty}}}$.

Proof. By an argument similar to [2, Corollary B.14], we can see that $\left(B_{d R}^{+} \otimes V\right)^{G_{K \infty}}$ is a finite-dimensional vector space over $\left(B_{d R}^{+}\right)^{G_{K_{\infty}}}$ and we can have a base $v_{1}, \ldots, v_{d}$ such that $v_{1}, \ldots, v_{d} \in(W(\mathscr{R}) \otimes V)^{G_{K_{\infty}}}$. Assume $e_{1}, \ldots, e_{d}$ are a base of $V / \mathbb{Q}_{p}$, and $\left(v_{1}, \ldots, v_{d}\right)=$ $\left(e_{1}, \ldots, e_{d}\right) A$ with $A \in \mathrm{GL}_{d}\left(\left(B_{d R}^{+} \otimes V\right)^{G_{K_{\infty}}}\right)$, then $\theta(\operatorname{det}(A)) \neq 0$.

For $k \geq 1,1 \leq i \leq d$, let $v_{i, k}=\sum_{m \in \mathbb{Z}} \pi^{-k m} \varphi^{m-1}\left(\eta([\varepsilon]-1)^{k+1} v_{i}\right)$, then this is a convergence sum and it converges to an element in $\left(B_{\text {crys }}^{+} \otimes V\right)^{G_{K \infty}}$. Consider that $v_{1, k} /$ $\varphi^{-1}(\eta([\varepsilon]-1))^{k+1}$ tends to $\varphi^{-1}\left(v_{i}\right)$ when $k \rightarrow \infty$, hence when $k \gg 1, v_{i, k} / \varphi^{-1}(\eta([\varepsilon]$ $-1))^{k+1}, \ldots, v_{d, k} / \varphi^{-1}(\eta([\varepsilon]-1))^{k+1}$ are linearly independent, hence $v_{1, k}, \ldots, v_{d, k}$ are linearly independent.

From $\varphi\left(t_{\pi}^{-k} v_{i, k}\right)=\left(t_{\pi}^{-k} v_{i, k}\right)$ we see that $t_{\pi}^{-k} v_{1, k}, \ldots, t_{\pi}^{-k} v_{d, k} \in\left(B_{\max }^{\varphi=1} \otimes V\right)^{G_{K \infty}}$ are a base of $\left(B_{d R} \otimes V\right)^{G_{K_{\infty}}}$ over $B_{d R}^{G_{K_{\infty}}}$.

LEMMA 2.9. $H^{1}\left(K_{\infty}, B_{d R} \otimes V\right)=0$ and $H^{1}\left(K_{\infty}, \mathscr{D}_{r}\left(\mathbb{Z}_{p}^{\times}, B_{\max }^{\varphi=1} \otimes V\right)\right)=0$.

Proof. Assume $\tau \rightarrow c_{\tau}$ is a cocycle from $G_{K_{\infty}} \rightarrow V$. From [2] we know that $H^{1}\left(K_{\infty}\right.$, $\left.W\left(\mathfrak{m}_{\mathscr{R}}\right) \otimes T\right)=0$, where $T \subset V$ is a Galois invariant lattice. Let $\omega=\left(\omega_{0}, \omega_{1}, \omega_{2}, \ldots\right) \in \mathscr{R}$, then $[\omega] c_{T} \in H^{1}\left(K_{\infty}, W\left(\mathfrak{m}_{\mathscr{R}}\right) \otimes T\right)=0$, so we can find a $c \in W(\mathscr{R}) \otimes V$ such that $[\omega] c_{T}=$ $(1-\tau) c$. From the ramification property, we can show that $[\omega]^{p-1} \equiv 0(\bmod p)$ in $A_{\text {crys }}$, hence $a=\sum_{n \in \mathbb{Z}}\left(\varphi^{n}([\omega]) / p^{n}\right)$ is convergent in $A_{\text {crys }}^{G_{K_{\infty}}}$ and $\varphi(a)=p a$. Let

$$
c^{\prime}=\frac{1}{p a} \sum \frac{\varphi^{n}}{p^{n}}\left([\omega]^{-1}(\varphi[\omega]) c\right),
$$

then it is easy to see that $(1-\tau) c^{\prime}=c_{\tau}$. Since $c^{\prime} \in\left(B_{\max }^{\varphi=1} \otimes V\right)$, this shows that the inclusion map $h^{1}\left(K_{\infty}, V\right) \rightarrow H^{1}\left(K_{\infty}, \mathrm{Fil}^{-1}\left(B_{\max }^{\varphi=1} \otimes V\right)\right)$ is the zero map. By similar arguments as 
in [2, Lemmas B.18 and B.19] we conclude that $H^{1}\left(K_{\infty}, B_{d R} \otimes V\right)=0, H^{1}\left(K_{\infty}, \mathscr{D}_{r}\left(\mathbb{Z}_{p}^{\times}, B_{\max }^{\varphi=1} \otimes\right.\right.$ $V))=0$.

LEMMA 2.10. For a $p$-adic representation $V$ of $G_{\mathbb{Q}_{p}}$, as a $\mathscr{D}_{0}\left(\mathbb{Z}_{p}^{\times}, \mathbb{Q}_{p}\right)$-module the following sequence is exact:

$$
0 \longrightarrow H^{1}\left(\Gamma, \mathscr{D}_{0}\left(\mathbb{Z}_{p}^{\times}, V\right)^{G_{K_{\infty}}}\right) \longrightarrow H^{1}\left(\mathbb{Q}_{p}, \mathscr{D}_{0}\left(\mathbb{Z}_{p}^{\times}, V\right)\right) \longrightarrow H^{1}\left(K_{\infty}, \mathscr{D}_{0}\left(\mathbb{Z}_{p}^{\times}, V\right)\right) \Gamma \longrightarrow 0
$$

Proof. The proof can be found in [2].

3. Perrin-Riou exponential map. For $I \subset \mathbb{Z}$, we have defined $L P^{I}, \mathscr{D}_{\mathrm{alg}}^{I}\left(\mathbb{Q}_{p}, A\right), \Phi$, and $\mathscr{F}_{\text {alg }}$ in [8, Section 4]. For a Galois module $A$, in this section, the Galois action on $\mathscr{D}_{\text {alg }}^{I}\left(\mathbb{Q}_{p}, A\right)$ is defined by

$$
\int f(x) \sigma(\mu)=\sigma\left(\int f(\kappa(\sigma) x) \mu\right)
$$

where $\mu \in \mathscr{D}_{\text {alg }}^{I}\left(\mathbb{Q}_{p}, A\right)$ and $\sigma \in G_{\mathbb{Q}_{p}}$. Recall that we have the following formulas:

$$
\begin{gathered}
\mathscr{F}_{\mathrm{alg}}\left(x^{k} 1_{a+p^{n} \mathbb{Z}_{p}}\right)(y)=p^{-n} \frac{k !}{(-t y)^{k}} \varepsilon(a y) 1_{p^{-n} \mathbb{Z}_{p}}(y), \\
\mathscr{F}_{\mathrm{alg}}\left(x^{k} 1_{\mathbb{Z}_{p}^{\times}}\right)=\frac{1}{(-t x)^{k}} \cdot 1_{\mathbb{Z}_{p}}-p^{-1} \frac{1}{(-t x)^{k}} 1_{p^{-1} \mathbb{Z}_{p}} .
\end{gathered}
$$

Proposition 3.1. For a de Rham representation $V$, if $\mu \in \mathscr{D}_{\mathrm{alg}}^{(-\infty, h-1]}\left(\mathbb{Q}_{p}, \Xi \otimes D(V)\right)^{\psi}$, then $\mathscr{F}_{\text {alg }}^{(h)}(\mu) \in \mathscr{D}_{\text {alg }}^{[1-h,+\infty)}\left(\mathbb{Q}_{p}, B_{d R} \otimes V\right)$ is fixed by $G_{\mathbb{Q}_{p}}$.

Proof. For $\sigma \in G_{\mathbb{Q}_{p}}$,

$$
\begin{aligned}
\int_{a+p^{n} \mathbb{Z}_{p}} x^{k} \sigma\left(\mathscr{F}_{\text {alg }}^{(h)}(\mu)\right)= & \sigma\left(\int_{\kappa(\sigma)^{-1} a+p^{n} \mathbb{Z}_{p}}(\kappa(\sigma) x)^{\left.k_{\mathscr{F}}^{(h)}(\mu)\right)}\right. \\
= & p^{-n}(k+h-1) ! \kappa(\sigma)^{k} \\
& \times\left(\sigma\left(\int_{p^{-n} \mathbb{Z}_{p}} \varepsilon\left(\kappa(\sigma)^{-1} a x\right)(-t x)^{-k} \mu\right)\right) \\
= & p^{-n}(k+h-1) !\left(\int_{p^{-n} \mathbb{Z}_{p}} \varepsilon(a x)(-t x)^{-k} \mu\right) \\
= & \int_{a+p^{n} \mathbb{Z}_{p}} x^{k \mathscr{F}_{\text {alg }}^{(h)}(\mu),}
\end{aligned}
$$

hence we have $\sigma\left(\mathscr{F}_{\text {alg }}^{(h)}(\mu)\right)=\mathscr{F}_{\text {alg }}^{(h)}(\mu)$.

Let exp denote the connecting map of the following exact sequence:

$$
0 \longrightarrow \mathscr{D}_{\mathrm{alg}}^{+}\left(\mathbb{Z}_{p}^{\times}, V\right) \longrightarrow \mathscr{D}_{\mathrm{alg}}^{+}\left(\mathbb{Z}_{p}^{\times}, B_{\max }^{\varphi=1} \otimes V\right) \longrightarrow \mathscr{D}_{\mathrm{alg}}^{+}\left(\mathbb{Z}_{p}^{\times}, B_{d R} / B_{d R}^{+} \otimes V\right) \longrightarrow 0
$$


Proposition 3.2. The following diagram is commutative:

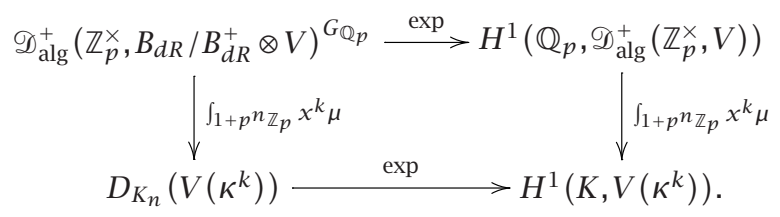

ProOF. For $\mu \in \mathscr{D}_{\mathrm{alg}}^{+}\left(\mathbb{Z}_{p}^{\times}, B_{d R} / B_{d R}^{+} \otimes V\right)^{G_{\mathbb{Q} p}}$, it is easy to see that $\int_{1+p^{n} \mathbb{Z}_{p}} x^{k} \mu \in$ $D_{K_{n}}\left(V\left(\kappa^{k}\right)\right)$ and for $\xi \in H^{1}\left(\mathbb{Q}_{p}, \mathscr{D}_{\text {alg }}^{+}\left(\mathbb{Z}_{p}^{\times}, V\right)\right), \int_{1+p^{n} \mathbb{Z}_{p}} x^{k} \xi \in H^{1}\left(K_{n}, V\left(\kappa^{k}\right)\right)$, then the proposition follows from the following commutative diagram:

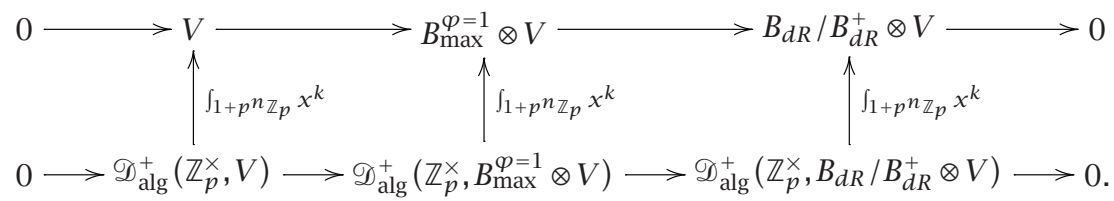

Now we define the Perrin-Riou exponential map $\mathbb{E}=\mathbb{E}_{h, V}$ as the composition of the following ones:

$$
\begin{aligned}
\mathscr{D}_{\text {alg }}^{-}\left(\mathbb{Q}_{p}, \Xi \otimes D(V)\right)^{\Phi=1, \psi} & \stackrel{\mathscr{F}_{\text {alg }}}{\longrightarrow} \mathscr{D}_{\mathrm{alg}}^{+}\left(\mathbb{Q}_{p}, B_{d R} \otimes V\right)^{G_{\mathbb{Q} p}} \\
& \stackrel{\text { Fil }^{<0}}{\longrightarrow} \mathscr{D}_{\mathrm{alg}}^{+}\left(\mathbb{Z}_{p}^{\times}, B_{d R} / B_{d R}^{+} \otimes V\right)^{G_{\mathbb{Q} p}} \\
& \stackrel{\exp }{\longrightarrow} H^{1}\left(\mathbb{Q}_{p}, \mathscr{D}_{\mathrm{alg}}^{+}\left(\mathbb{Z}_{p}^{\times}, V\right)\right) .
\end{aligned}
$$

The invariance of $\Phi \mu=\mu$ gives the following identity:

$$
\int_{p^{-n} \mathbb{Z}_{p}} f(x) \frac{1}{(-t x)^{k}} \mu=\varphi^{-n}\left(\int_{\mathbb{Z}_{p}} f\left(\frac{x}{p^{n}}\right) \frac{\mu}{(-t x)^{k}}\right)
$$

for all $n \in \mathbb{Z}, k \geq 0$, and $f$ a local constant function with compact support $p^{-n} \mathbb{Z}_{p}$.

THEOREM 3.3. The integrals of the exponential map give

$$
\begin{aligned}
& \int_{\mathbb{Z}_{p}^{\times}} x^{k} \mathbb{E}_{h, V}(\mu)=(k+h-1) ! \exp _{V\left(\kappa^{k}\right)}\left((1-\varphi)^{-1}\left(1-p^{-1} \varphi^{-1}\right) \int_{\mathbb{Z}_{p}^{\times}} \frac{\mu}{(-t x)^{k}}\right), \\
& \int_{a+p^{n} \mathbb{Z}_{p}} x^{k} \mathbb{E}_{h, V}(\mu)=(k+h-1) ! \exp _{V\left(\kappa^{k}\right)}\left(\frac{\varphi^{-n}}{p^{n}}\left(\int_{\mathbb{Z}_{p}} \varepsilon\left(\frac{a x}{p^{n}}\right) \cdot \frac{\mu}{(-t x)^{k}}\right)\right) .
\end{aligned}
$$


Proof. By formulas (3.2) and (3.3), we have

$$
\begin{aligned}
\int_{\mathbb{Z}_{p}^{\times}} x^{k} \mathscr{F}_{\mathrm{alg}}^{(h)}(\mu) & =\int_{\mathbb{Q}_{p}} \mathscr{F}_{\mathrm{alg}}^{(h)}\left(x^{k} 1_{\mathbb{Z}_{p}^{\times}}\right) \mu \\
& =\int_{\mathbb{Q}_{p}}(k+h-1) !\left(\frac{1}{(-t x)^{k}} 1_{\mathbb{Z}_{p}}-p^{-1} \frac{1}{(-t x)^{k}} 1_{p^{-1} \mathbb{Z}_{p}}\right) \mu \\
& =(k+h-1) !(1-\varphi)^{-1}\left(1-p^{-1} \varphi^{-1}\right)\left(\int_{\mathbb{Z}_{p}^{\times}} \frac{\mu}{(-t x)^{k}}\right), \\
\int_{a+p^{n} \mathbb{Z}_{p}} x^{k \mathscr{F}_{\mathrm{alg}}^{(h)}(\mu)} & =\int_{\mathbb{Q}_{p}} \mathscr{F}_{\mathrm{alg}}^{(h)}\left(x^{k} 1_{a+p^{n} \mathbb{Z}_{p}}\right) \mu \\
& =\int_{\mathbb{Q}_{p}}(k+h-1) ! p^{-n} \cdot \frac{1}{(-t x)^{k}} \varepsilon(a x) 1_{p^{n} \mathbb{Z}_{p}} \\
& =(k+h-1) ! \frac{\varphi^{-n}}{p^{n}}\left(\int_{\mathbb{Z}_{p}} \varepsilon\left(\frac{a x}{p^{n}}\right) \frac{\mu}{(-t x)^{k}}\right) .
\end{aligned}
$$

Hence, by Proposition 1.2, we have

$$
\begin{aligned}
\int_{\mathbb{Z}_{p}^{\times}} x^{k} \mathbb{E}_{h, V}(\mu) & =\exp _{V\left(\kappa^{k}\right)}\left(\int_{\mathbb{Q}_{p}} \mathscr{F}_{\mathrm{alg}}\left(x^{k} \cdot 1_{\mathbb{Z}_{p}^{\times}}\right) \mu\right) \\
& =\exp _{V\left(\kappa^{k}\right)}\left((k+h-1) !(1-\varphi)^{-1}\left(1-p^{-1} \varphi^{-1}\right) \int_{\mathbb{Z}_{p}^{\times}} \frac{\mu}{(-t x)^{k}}\right), \\
\int_{a+p^{n} \mathbb{Z}_{p}} x^{k} \mathbb{E}_{h, V}(\mu) & =\exp _{V\left(\kappa^{k}\right)}\left(\int_{\mathbb{Q}_{p}} \mathscr{F}_{\mathrm{alg}}\left(x^{k} 1_{a+p^{n} \mathbb{Z}_{p}} \mu\right)\right) \\
& =\exp _{V\left(\kappa^{k}\right)}\left((k+h-1) ! \frac{\varphi^{-n}}{p^{n}}\left(\int_{\mathbb{Z}_{p}} \varepsilon\left(\frac{a x}{p^{n}}\right) \frac{\mu}{(-t x)^{k}}\right)\right) .
\end{aligned}
$$

LEMMA 3.4. For a de Rham representation $V$ such that $\mathrm{Fil}^{-1} D(V)=D(V), \mu \in$ $\mathscr{D}_{\mathrm{alg}}^{(-\infty, 0]}\left(\mathbb{Q}_{p}, \Xi \otimes D(V)\right)^{\Phi=1, \psi}, a \in \mathbb{Z}_{p}^{\times}, n \geq 1$, and $j \geq 0, \int_{a+p^{n} \mathbb{Z}_{p}}(x-a)^{j} \mathbb{E}_{h, V}(\mu)$ restricted to $H^{1}\left(K_{\infty}, \mathscr{D}_{\mathrm{alg}}^{[0, \infty)}\left(\mathbb{Z}_{p}^{\times}, V\right)\right)$ is represented by the cocycle

$$
\tau \longrightarrow(\tau-1) \operatorname{Eul}_{B}\left(\widetilde{\beta}_{a, n, j}(\mu)\right),
$$

where

$$
\tilde{\beta}_{a, n, j}(\mu)=j ! p^{n j}(-t)^{j} \frac{\varphi^{-n}}{p^{n}} \int_{\mathbb{Z}_{p}^{\times}}[\varepsilon]^{a x} \frac{\mu}{x^{j}} .
$$

Proof. We first consider

$$
\int_{a+p^{n} \mathbb{Z}_{p}} x^{k} \mathbb{E}_{1, V}(\mu)=\exp _{V\left(\kappa^{k}\right)}\left(k ! \frac{\varphi^{-n}}{p^{n}} \int_{\mathbb{Z}_{p}} \varepsilon\left(\frac{a x}{p^{n}}\right) \frac{\mu}{(-t x)^{k}}\right) .
$$


Put

$$
\begin{aligned}
& \gamma_{a, n, k}(\mu)=k ! \frac{\varphi^{-n}}{p^{n}} \int_{\mathbb{Z}_{p}} \varepsilon\left(\frac{a x}{p^{n}}\right) \frac{\mu}{(-t x)^{k}}, \\
& \tilde{\gamma}_{a, n, k}(\mu)=k ! \frac{\varphi^{-n}}{p^{n}} \int_{\mathbb{Z}_{p}}\left[\varepsilon^{a x}\right] \sum_{i=0}^{k} \frac{(-a t x)^{i}}{i !} \frac{\mu}{(-t x)^{k}} .
\end{aligned}
$$

By the formula $\varepsilon\left(1 / p^{n}\right)=\left[\varepsilon_{n}\right] \exp \left(-t / p^{n}\right)$, we have

$$
\varepsilon\left(\frac{x}{p^{n}}\right) \equiv\left[\varepsilon_{n}^{x}\right]\left(\sum_{i=0}^{k} \frac{1}{i !}\left(\frac{-t x}{p^{n}}\right)^{i}\right)=\varphi^{-n}\left(\left[\varepsilon^{x}\right] \sum_{i=0}^{k} \frac{(-t x)^{i}}{i !}\right) \bmod \left(\mathrm{Fil}^{k+1} B_{d R}\right) .
$$

Let

$$
\begin{aligned}
\delta_{a, n, k}(x) & =\varepsilon\left(\frac{a x}{p^{n}}\right)-\varphi^{-n}\left(\left[\varepsilon^{a x}\right] \sum_{i=0}^{k} \frac{(-a t x)^{i}}{i !}\right) \in \mathrm{Fil}^{k+1} B_{d R} \\
& =\varphi^{-n}\left(\varepsilon\left(\frac{a x}{p^{n}}\right)-\left[\varepsilon^{a x}\right] \sum_{i=0}^{k} \frac{(-a t x)^{i}}{i !}\right),
\end{aligned}
$$

then

$$
\begin{aligned}
\tilde{\gamma}_{a, n, k}(\mu)-\gamma_{a, n, k}(\mu) & =k ! \frac{\varphi^{-n}}{p^{n}} \int_{\mathbb{Z}_{p}} \varphi^{n}\left(\delta_{a, n, k}\right) \frac{\mu}{(-t x)^{k}} \\
& \in B_{d R}^{1} \otimes D(V) \subset B_{d R}^{1} \otimes\left(B_{d R}^{-1} \otimes V\right) \subset B_{d R}^{+} \otimes V .
\end{aligned}
$$

Hence, $\tilde{\gamma}_{a, n, k}(\mu) \in B_{\max } \otimes V$ is a lifting of $\gamma_{a, n, k}$ under the projection map $B_{\max } \rightarrow$ $B_{d R} / B_{d R}^{+}$. By [2, Lemma 0.5.1], we see that the above cohomology class $\exp _{V\left(\kappa^{k}\right)}\left(\gamma_{a, n, k}(\mu)\right)$ is represented by the cocycle $\tau \rightarrow(1-\tau) \operatorname{Eul}_{B}\left((1-\varphi) \tilde{\gamma}_{a, n, k}(\mu)\right)$. Moreover,

$$
\begin{aligned}
(1-\varphi) \tilde{\gamma}_{a, n, k}(\mu) & =(1-\varphi) k ! \frac{\varphi^{-n}}{p^{n}}\left(\int_{\mathbb{Z}_{p}}\left[\varepsilon^{a x}\right] \sum_{i=0}^{k} \frac{(-a t x)^{i}}{i !} \frac{\mu}{(-t x)^{k}}\right) \\
& =k ! \frac{\varphi^{-n}}{p^{n}}\left(\int_{\mathbb{Z}_{p}^{x}}\left[\varepsilon^{a x}\right] \sum_{i=0}^{k} \frac{(-a t x)^{i}}{i !} \frac{\mu}{(-t x)^{k}}\right)
\end{aligned}
$$

since

$$
\varphi\left(\int_{\mathbb{Z}_{p}}\left[\varepsilon^{a x}\right] \sum_{i=0}^{k} \frac{(-a t x)^{i}}{i !} \frac{\mu}{(-t x)^{i}}\right)=\int_{\mathbb{Z}_{p}}\left[\varepsilon^{a x}\right] \sum_{i=0}^{k} \frac{(-a t x)^{i}}{i !} \frac{1}{(-t x)^{k}} \Phi \mu,
$$

and $\Phi\left(\mu \cdot 1_{\mathbb{Z}_{p}}\right)=1_{p \mathbb{Z}_{p}} \cdot \mu$.

Hence, for $a \in \mathbb{Z}_{p}^{\times}, n \geq 1, \int_{a+p^{n} \mathbb{Z}_{p}} x^{k} \mathbb{E}_{1, V}(\mu)$ is represented by

$$
\tau \longrightarrow(\tau-1) \operatorname{Eul}_{B}\left(\gamma_{a, n, k}^{*}(\mu)\right),
$$


where

$$
\gamma_{a, n, k}^{*}(\mu)=k ! \frac{\varphi^{-n}}{p^{n}}\left(\int_{\mathbb{Z}_{p}^{\times}}\left[\varepsilon^{a x}\right] \sum_{i=0}^{k} \frac{(-a t x)^{i}}{i !} \frac{v}{(-t x)^{k}}\right) .
$$

Therefore,

$$
\begin{aligned}
\int_{a+p^{n} \mathbb{Z}_{p}}(x-a)^{j} \mathbb{E}_{1, V}(\mu) & =\sum_{k=0}^{j} \int_{a+p^{n} \mathbb{Z}_{p}}\left(\begin{array}{l}
j \\
k
\end{array}\right)(-a)^{j-k} x^{k} \mathbb{E}_{1, V}(\mu) \\
& =\sum_{k=0}^{j}\left(\begin{array}{l}
j \\
k
\end{array}\right)(-a)^{j-k} \int_{a+p^{n} \mathbb{Z}_{p}} x^{k} \mathbb{E}_{1, V}(\mu)
\end{aligned}
$$

is represented by

$$
\begin{aligned}
\tau & \longrightarrow(\tau-1) \sum_{k=0}^{j}\left(\begin{array}{l}
j \\
k
\end{array}\right)(-a)^{j-k} \operatorname{Eul}_{B}\left(\gamma_{a, n, k}^{*}(\mu)\right) \\
= & (\tau-1) \operatorname{Eul}_{B}\left(\sum_{k=0}^{j}\left(\begin{array}{l}
j \\
k
\end{array}\right)(-a)^{j-k} k ! \frac{\varphi^{-n}}{p^{n}} \int_{\mathbb{Z}_{p}^{\times}}\left[\varepsilon^{a x}\right] \sum_{i=0}^{k} \frac{(-a t x)^{i}}{i !} \frac{\mu}{(-t x)^{k}}\right) \\
= & (\tau-1) \operatorname{Eul}_{B}\left(\frac{\varphi^{-n}}{p^{n}} \int_{\mathbb{Z}_{p}^{\times}}\left(e^{a t x} \cdot \sum_{k=0}^{j}\left(k !\left(\begin{array}{l}
j \\
k
\end{array}\right)(a t x)^{j-k}\left(\sum_{k=0}^{k} \frac{(-a t x)^{i}}{i !}\right)\right)\right)\right. \\
& \left.\times \frac{\mu}{(-t x)^{j}}\right) \\
= & (\tau-1) \operatorname{Eul}_{B}\left(\frac{\varphi^{-n}}{p^{n}} \int_{\mathbb{Z}_{p}^{\times}}\left(j !\left[\varepsilon^{a x}\right]\right) \frac{\mu}{(-t x)^{j}}\right),
\end{aligned}
$$

and $\int_{a+p^{n} \mathbb{Z}_{p}}(x-a)^{j} \mathbb{E}_{1, V}(\mu)$ is represented by

$$
\tau \longrightarrow(\tau-1) \operatorname{Eul}_{B}\left(j ! p^{n j}(-t)^{-j} \frac{\varphi^{-n}}{p^{n}} \int_{\mathbb{Z}_{p}^{x}}\left[\varepsilon^{a x}\right] \frac{\mu}{x^{j}}\right),
$$

and the lemma follows.

LEMMA 3.5. The following diagram is commutative:

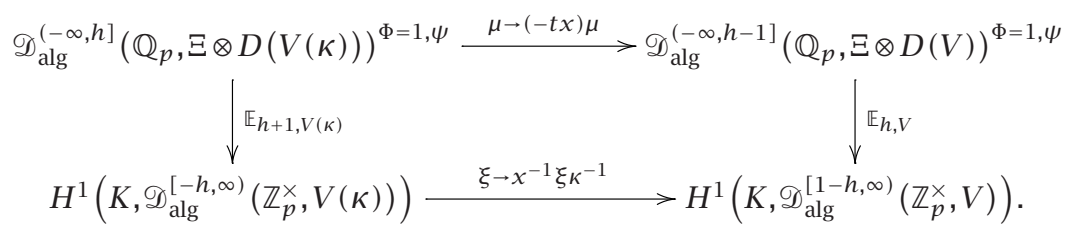


Proof. First note that all the maps are well defined. For $\mu \in \mathscr{D}_{\text {alg }}^{(-\infty, h]}\left(\mathbb{Q}_{p}, \Xi \otimes\right.$ $D(V(\kappa)))^{\psi},(-t x) \mu \in \mathscr{D}_{\text {alg }}^{(-\infty, h-1]}\left(\mathbb{Q}_{p}, \Xi \otimes D(V)\right)^{\psi}$,

$$
\begin{aligned}
& \int_{\mathbb{Z} \times \vec{p}} x^{k} \mathbb{E}_{h, V}((-t x) \mu) \\
& \quad=(k+h-1) ! \exp _{V\left(\kappa^{k}\right)}\left((1-\varphi)^{-1}\left(1-p^{-1} \varphi^{-1}\right) \int_{\mathbb{Z}_{p}^{\times}} \frac{\mu}{(-t x)^{k-1}}\right), \\
& \int_{\mathbb{Z}_{p}^{\times}} x^{k} \cdot x^{-1} \mathbb{E}_{h+1, V(\kappa)}(\mu) \\
& \quad=(k+h-1) ! \exp _{V\left(\kappa^{k}\right)}\left((1-\varphi)^{-1}\left(1-p^{-1} \varphi^{-1}\right) \int_{\mathbb{Z}_{p}^{\times}} \frac{\mu}{(-t x)^{k-1}}\right),
\end{aligned}
$$

hence the lemma follows.

THEOREM 3.6. Assume $V$ is a crystalline representation and $h \in \mathbb{Z}$ such that $\mathrm{Fil}^{-h} D(V)$ $=D(V)$. For $\mu \in \widetilde{D}_{\text {temp }}\left(\mathbb{Q}_{p}, \Xi \otimes D(V)\right)^{\Phi=1, \psi}$, under the map

$$
\begin{aligned}
\mathscr{D}_{\mathrm{alg}}^{(-\infty, h-1]}\left(\mathbb{Q}_{p}, \Xi \otimes D(V)\right)^{\Phi=1, \psi} & \longrightarrow H^{1}\left(\mathbb{Q}_{p}, \mathscr{D}_{\mathrm{alg}}^{[1-h, \infty)}\left(\mathbb{Z}_{p}^{\times}, V\right)\right) \\
& \stackrel{\text { res }}{\longrightarrow} H^{1}\left(K_{\infty}, \mathscr{D}_{\mathrm{alg}}^{[1-h, \infty)}\left(\mathbb{Z}_{p}^{\times}, V\right)\right)^{\Gamma},
\end{aligned}
$$

the image is in $H^{1}\left(K_{\infty}, \mathscr{D}_{\text {temp }}\left(\mathbb{Z}_{p}^{\times}, V\right)\right)^{\Gamma}$ with $\Gamma=\operatorname{Gal}\left(K_{\infty} / \mathbb{Q}_{p}\right)$.

Proof. Since $x^{1-h} \mathbb{E}_{1, V\left(\kappa^{1-h)}\right.}\left((-t x)^{h-1} \mu\right)=\mathbb{E}_{h, V}(\mu)$, by Lemma 3.5, replacing $V$ by $V(1-h)$ and $\mu$ by $(-t x)^{h-1} \mu$, we can assume $h=1$. From Lemma 3.5 for $a \in \mathbb{Z}_{p}^{\times}, n \geq 1$, and $j \geq 0, \int_{a+p^{n} \mathbb{Z}_{p}}(x-a)^{j} \mathbb{E}_{1, V}(\mu)$ is represented by the cocycle

$$
\tau \longrightarrow(\tau-1) \operatorname{Eul}_{B}\left(j ! p^{n j}(-t)^{-j} \frac{\varphi^{-n}}{p^{n}} \int_{\mathbb{Z}_{p}^{x}}\left[\varepsilon^{a x}\right] \frac{\mu}{x^{j}}\right) .
$$

From [8, Lemma 14], we know that if $\mu$ is of order $r$, then $\mu / x^{j}$ is also of order $r$. From Proposition 2.4, we know that $\int_{\mathbb{Z}_{p}^{\times}}\left[\varepsilon^{a x}\right]\left(\mu / x^{j}\right)$ has order $r+r(V)$, that is,

$$
p^{[n(r+r(V)+1)]} \varphi^{-n} \int_{\mathbb{Z}_{p}^{\times}}\left[\varepsilon^{a x}\right] \frac{\mu}{x^{j}}
$$

is $r$-bounded. Hence, $p^{[n(r+r(V)+1)]} \int_{a+p^{n} \mathbb{Z} p}(x-a)^{j} \mathbb{E}_{1, V}(\mu)$ represented by

$$
\tau \longrightarrow(\tau-1) \operatorname{Eul}_{B}\left(j !(-t)^{-j}\left(p^{[n(r+r(V)+1)]} \varphi^{-n} \int_{\mathbb{Z}_{p}^{x}}\left[\varepsilon^{a x}\right] \frac{\mu}{x^{j}}\right)\right)
$$

is $(r+r(V))$-bounded. Hence, the image is in $H^{1}\left(K_{\infty}, \mathscr{D}_{r+r(V)+1}\left(\mathbb{Z}_{p}^{\times}, V\right)\right)^{\Gamma}$.

4. The logarithmic map. The proof of Theorem 3.6 suggests that $\mathscr{F}_{\text {cont }}(\mu)$ has some deep arithmetic meaning for the distribution $\mu$. In this section, we construct a logarithmic map on the cohomology side, which is related to $\mathscr{F}_{\text {cont }}(\mu)$.

Suppose $V$ is a de Rham representation. The $\mathbb{Q}_{p}$-space $\cup_{n \in \mathbb{N}}\left(B_{\max }^{\varphi=1} \otimes V\left(\kappa^{-i}\right)\right)^{G_{K n}} \subset$ $\cup_{n \in \mathbb{N}}\left(B_{\max } \otimes V\right)^{G_{K n}}$ has dimension $\operatorname{dim}_{\mathbb{Q}_{p}} V$, so $\cup_{n \in \mathbb{N}}\left(B_{\max }^{\varphi=1} \otimes V\left(\kappa^{-i}\right)\right)^{G_{K n}}$ is a closed 
subspace of $B_{\max }^{\varphi=1} \otimes V$. Let $W_{i}$ be one supplement. Suppose $h \in \mathbb{Z}, h \geq 1, \mu \in H^{1}\left(\mathbb{Q}_{p}\right.$, $\left.\mathscr{D}_{h^{-}}\left(\mathbb{Z}_{p}^{\times}, V\right)\right)$, and $\tau \rightarrow \mu_{\tau}$ is a 1-cocycle representation of $\mu$. Suppose $\mu$ has the property $\int_{1+p^{n} \mathbb{Z}_{p}} x^{-i} \mu \in H_{e}^{1}\left(K_{n}, V\left(\kappa^{-i}\right)\right)=\operatorname{ker}\left\{H^{1}\left(K_{n}, V\left(\kappa^{-i}\right)\right) \rightarrow H^{1}\left(K_{n}, B_{\max }^{\varphi=1} \otimes V\left(\kappa^{-i}\right)\right)\right\}$. For such a $\mu$, there is a $c_{n, i} \in B_{\max }^{\varphi=1} \otimes V\left(\kappa^{-i}\right)$ such that

$$
\left(1-\kappa(\sigma)^{-i} \sigma\right) c_{n, i}=\int_{1+p^{n} \mathbb{Z}_{p}} x^{-i} \mu_{\sigma} .
$$

The choice of $W_{i}$ makes it possible to choose $c_{n, i} \in W_{i}$ uniquely for $n \gg 1$.

THEOREM 4.1. Suppose $V$ is a de Rham representation of $G_{\mathbb{Q}_{p}}, h \geq 1$ is an integer, and $\mu \in H^{1}\left(\mathbb{Q}_{p}, \mathscr{D}_{h^{-}}\left(\mathbb{Z}_{p}^{\times}, V\right)\right)$ such that $\int_{1+p^{n} \mathbb{Z}_{p}} x^{-i} \mu \in H_{e}^{1}\left(K_{n}, V\left(\kappa^{-i}\right)\right)$ for $n \geq 1,0 \leq i \leq h-1$. Choose $c_{n, i} \in W_{i}$ such that

$$
\left(1-\kappa(\sigma)^{-i} \sigma\right) c_{n, i}=\int_{1+p^{n} \mathbb{Z}_{p}} x^{-i} \mu,
$$

then the sequence $(1 /(h-1) !) p^{n} \sum_{i=0}^{h-1}\left(\begin{array}{c}h-1 \\ i\end{array}\right) c_{n, i}$ converges to an element in $\left(B_{\max }^{\varphi=1} \otimes\right.$ $V)^{G_{K \infty}}$ which is denoted by $\mathbb{L}_{h, V}(\mu)$.

Proof. By Lemma $2.9, H^{1}\left(K_{\infty}, \mathscr{D}_{h^{-}}\left(\mathbb{Z}_{p}^{\times}, B_{\max }^{\varphi=1} \otimes V\right)\right)=0$, the inflation-restriction sequence gives the following isomorphism:

$$
H^{1}\left(\mathbb{Q}_{p}, \mathscr{D}_{h^{-}}\left(\mathbb{Z}_{p}^{\times}, B_{\max }^{\varphi=1} \otimes V\right)\right) \stackrel{\sim}{\longrightarrow} H^{1}\left(\Gamma, \mathscr{D}_{h^{-}}\left(\mathbb{Z}_{p}^{\times},\left(B_{\max }^{\varphi=1} \otimes V\right)\right)^{G_{K_{\infty}}}\right) .
$$

Let $\mu^{\prime} \in H^{1}\left(\Gamma, \mathscr{D}_{h^{-}}\left(\mathbb{Z}_{p}^{\times},\left(B_{\max }^{\varphi=1} \otimes V\right)^{G_{K \infty}}\right)\right)$ correspond to the image of $\mu$ under the map $H^{1}\left(\mathbb{Q}_{p}, \mathscr{D}_{h^{-}}\left(\mathbb{Z}_{p}^{\times}, V\right)\right) \rightarrow H^{1}\left(\mathbb{Q}_{p}, \mathscr{D}_{h^{-}}\left(\mathbb{Z}_{p}^{\times}, B_{\max }^{\varphi=1} \otimes V\right)\right)$. Then $\mu-\mu^{\prime}$ is a coboundary in $H^{1}\left(\mathbb{Q}_{p}\right.$, $\left.\mathscr{D}_{h^{-}}\left(\mathbb{Z}_{p}^{\times}, B_{\max }^{\varphi=1} \otimes V\right)\right)$, that is, there exists $v \in \mathscr{D}_{h^{-}}\left(\mathbb{Z}_{p}^{\times}, B_{\max }^{\varphi=1} \otimes V\right)$ such that $\mu_{\sigma}-\mu_{\sigma}^{\prime}=(1-$ $\sigma) \nu$. Let $u_{n, i}=c_{n, i}-\int_{1+p^{n} \mathbb{Z}_{p}} x^{-i} v$, then $u_{n, i} \in\left(B_{\max }^{\varphi=1} \otimes V\left(\kappa^{-i}\right)\right)^{G_{K_{\infty}}}$ and $\left(1-\kappa(\sigma)^{-i} \sigma\right) u_{n, i}$ $=\int_{1+p^{n} \mathbb{Z}_{p}} x^{-i} \mu_{\sigma}^{\prime}$ for $\sigma \in G_{K_{n}}$, and we have

$$
\begin{gathered}
\lim _{n \rightarrow \infty}\left(p^{n} \sum_{i=0}^{h-1}(-1)^{i}\left(\begin{array}{c}
h-1 \\
i
\end{array}\right) c_{n, i}-p^{n} \sum_{i=0}^{h-1}(-1)^{i}\left(\begin{array}{c}
h-1 \\
i
\end{array}\right) u_{n, i}\right) \\
=\lim _{n \rightarrow \infty} p^{n} \int_{1+p^{n} \mathbb{Z} p}\left(1-x^{-1}\right)^{h-1} v=0 .
\end{gathered}
$$

Here, again, we use [8, Lemma 14]. Note that there exists a $k$ such that $u_{n, i} \in\left(t^{-k} B_{\max }^{+}\right)^{\varphi=1}$ $\otimes V\left(\kappa^{-i}\right)$ (which does not depend on $\left.n, i\right)$ [2]. The theorem will follow from the following lemma.

LEMmA 4.2. Suppose $\mu \in H^{1}\left(\Gamma, \mathscr{D}_{h^{-}}\left(\mathbb{Z}_{p}^{\times},\left(B_{\max }^{\varphi=1} \otimes V\right)^{G_{K_{\infty}}}\right)\right)$ and $k \in \mathbb{N}$ such that for $n \geq 1$ and $0 \leq i \leq h-1$, the image $\int_{1+p^{n} \mathbb{Z}_{p}} x^{-i} \mu$ is zero in $H^{1}\left(\Gamma_{n},\left(\text { Fil }^{-k} B_{\max }^{\varphi=1} \otimes V\right)^{G_{K_{\infty}}}\right)$. Suppose

$$
\left(\mathrm{Fil}^{-k} B_{\max }^{\varphi=1} \otimes V\right)^{G_{K \infty}}=\cup_{n=0}^{+\infty}\left(\mathrm{Fil}^{-k} B_{\max }^{\varphi=1} \otimes V\left(\kappa^{-i}\right)\right)^{G_{K n}} \oplus W_{i}^{\prime}
$$


is a direct summand decomposition (since the first component is closed). Suppose $\sigma \rightarrow$ $\mu_{\sigma}$ is a cocycle representation of $\mu$, take $u_{n, i} \in W_{i}^{\prime}$ such that $\int_{1+p^{n} \mathbb{Z}_{p}} x^{-i} \xi_{\sigma}=(1-$ $\left.\kappa^{-i}(\sigma) \sigma\right) u_{n, i}$ for all $\sigma \in G_{K_{n}}$, then the sequence $v_{n}=p^{n} \sum_{i=0}^{h-1}(-1)^{i}\left(\begin{array}{c}h-1 \\ i\end{array}\right) u_{n, i}$ converges to an element in $\left(\mathrm{Fil}^{-k} B_{\max }^{\varphi=1} \otimes V\right)^{G_{K_{\infty}}}$.

Proof. The cocycles satisfy $\mu_{\sigma^{-1} \tau}=\mu_{\sigma^{-1}}+\sigma^{-1} \mu_{\tau}=\mu_{\tau}+\tau \mu_{\sigma^{-1}}$, and this means that

$$
\begin{aligned}
\sigma^{-1} \mu_{\tau} & =\mu_{\tau}+(\tau-1) \mu_{\sigma^{-1}}, \\
\int_{1+p^{n} \mathbb{Z}_{p}} x^{-i} \sigma^{-1} \mu_{\tau} & =\sigma^{-1}\left(\int_{\kappa(\sigma)+p^{n} \mathbb{Z}_{p}} \kappa^{i}(\sigma) x^{-i} \mu_{\tau}\right) .
\end{aligned}
$$

This gives

$$
\begin{aligned}
\int_{\kappa(\sigma)+p^{n} \mathbb{Z}_{p}} x^{-i} \mu_{\tau} & =\kappa(\sigma)^{-i} \sigma\left(\int_{1+p^{n} \mathbb{Z}_{p}} x^{-i} \cdot \sigma^{-1} \mu_{\tau}\right) \\
& =t_{\pi}^{i} \cdot \sigma\left(\int_{1+p^{n} \mathbb{Z}_{p}}\left(t_{\pi} x\right)^{-i} \cdot \sigma^{-1} \mu_{\tau}\right) \\
& =t_{\pi}^{i}(1-\tau) \sigma\left(t_{\pi}^{-i} u_{n, i}-\int_{1+p^{n} \mathbb{Z}_{p}}\left(t_{\pi} x\right)^{-i} \mu_{\sigma^{-1}}\right)
\end{aligned}
$$

by

$$
\begin{aligned}
t_{\pi}^{-i}(1-\tau) u_{n-1, i} & =t_{\pi}^{-i} \cdot \sum_{\sigma \in \Gamma_{n-1} / \Gamma_{n}} \int_{\kappa(\sigma)+p^{n} \mathbb{Z}_{p}} x^{-i} \mu_{\sigma} \\
& =(1-\tau) \sum_{\sigma \in \Gamma_{n-1} / \Gamma_{n}}\left(\sigma\left(t_{\pi}^{-i} u_{n, i}\right)-\sigma\left(\int_{1+p^{n} \mathbb{Z}_{p}}\left(t_{\pi} x\right)^{-i} \mu_{\sigma^{-1}}\right)\right) .
\end{aligned}
$$

From the choice of $W_{i}^{\prime}$, we can cancel $(1-\tau)$ and get

$$
\begin{aligned}
v_{n}-v_{n-1}=p^{n-1} \sum_{i=0}^{h-1}(-1)^{i}\left(\begin{array}{c}
h-1 \\
i
\end{array}\right) t_{\pi}^{i} & \left(\sum_{\sigma \in \Gamma_{n-1} / \Gamma_{n}}(\sigma-1) t_{\pi}^{-i} u_{n, i}\right. \\
& \left.-\sigma\left(\int_{1+p^{n} \mathbb{Z}_{p}}\left(t_{\pi} x\right)^{-i} \mu_{\sigma^{-1}}\right)\right) .
\end{aligned}
$$

The sum

$$
\begin{gathered}
p^{n} \sum_{i=0}^{h-1}(-1)^{i}\left(\begin{array}{c}
h-1 \\
i
\end{array}\right) t_{\pi}^{i} \sigma\left(\int_{1+p^{n} \mathbb{Z}_{p}}\left(t_{\pi} x\right)^{-i} \mu_{\sigma^{-1}}\right) \\
=p^{n} \sigma\left(\int_{1+p^{n} \mathbb{Z}_{p}}\left(1-\kappa(\sigma)^{-1} x\right)^{h-1} \mu_{\sigma^{-1}}\right) \rightarrow 0
\end{gathered}
$$

as $\kappa(\sigma) \in 1+p^{n-1} \mathbb{Z}_{p}, \mu_{\sigma^{-1}} \rightarrow \mu_{1}$ which is of order $1^{-}$. Hence, to show that $v_{n}$ is a Cauchy sequence, we only need to show that for $\sigma_{n} \in \Gamma_{n-1}$ the sequence

$$
p^{n} \sum_{i=0}^{h-1}(-1)^{i}\left(\begin{array}{c}
h-1 \\
i
\end{array}\right)\left(\kappa\left(\sigma_{n}\right)^{i} \sigma_{n}-1\right) u_{n, i}
$$

tends to zero. 
CASE $1(h=1)$. This is equivalent to showing that

$$
\lim _{n \rightarrow+\infty} p^{n}\left(\sigma_{n}-1\right) u_{n, i}=0
$$

Put $T_{\sigma}=\sum_{j=0}^{p-1} \sigma^{j}$, then

$$
T_{\sigma_{n}}\left(\sigma_{n}-1\right) u_{n, 0}=\left(\sigma_{n}^{p}-1\right) u_{n, 0}=-\int_{1+p^{n} \mathbb{Z}_{p}} \mu_{\sigma_{n}^{p}}
$$

Since $\mu_{\sigma_{n}^{p}}$ has order $1^{-}$, we have

$$
\lim _{n \rightarrow \infty} p^{n} \int_{1+p^{n} \mathbb{Z}_{p}} \mu_{\sigma_{n}^{p}}=0
$$

hence

$$
\lim _{n \rightarrow \infty} p^{n} T_{\sigma_{n}}\left(\sigma_{n}-1\right)\left(u_{n, 0}\right)=0
$$

The theorem follows from the following proposition.

Proposition 4.3. There exists a constant $C>0$ such that, for all $n \in \mathbb{N}, \sigma \in \Gamma_{n}$, $F \in\left(\mathrm{Fil}^{-k} B_{\max } \otimes V\right)^{G_{K n}}$,

$$
\left\|T_{\sigma}(F)\right\| \geq C\|F\|
$$

where $\|\cdot\|$ is the norm on $B_{\max }$.

In the following we will develop several lemmas to prove the above proposition.

LEMMA 4.4. There exist $a \zeta \in \mathbb{Z}_{p}^{\times}$and a $B_{\max }$-morphism $g: B_{\max } \otimes V \rightarrow B_{\max }^{d}$ such that $g\left(B_{\max }^{\varphi=1} \otimes V\right)^{G_{K \infty}}$ is contained in $\left(\left(B_{\max }^{\varphi=\zeta}\right)^{G_{K \infty}}\right)^{d}$, where $d=\operatorname{dim}_{\mathbb{Q}_{p}} V$.

Proof. Suppose $e_{1}, \ldots, e_{d} \in\left(B_{\max }^{\varphi=1} \otimes V\right)^{G_{K \infty}}$ such that they are a base of $B_{d R} \otimes V$ over $B_{d R}$ by Lemma 2.8. Assume $v_{1}, \ldots, v_{d}$ are a base of $V / \mathbb{Q}_{p}$ and assume $e_{i}=\sum_{j=1}^{d} a_{i j} v_{j}$, then $a_{i j} \in B_{\max }^{\varphi=1}$, the $\sigma$ action gives $\operatorname{det}(\sigma) \cdot \operatorname{det}\left(\sigma\left(a_{i j}\right)\right)=\operatorname{det}\left(a_{i j}\right)$. Consider the onedimensional representation $\operatorname{det}_{\mathbb{Q}_{p}}(V)$ which has a base $e=v_{1} \wedge \cdots \wedge v_{d}$, then the above formula gives $e_{1} \wedge \cdots \wedge e_{d}=\left(\operatorname{det}\left(a_{i j}\right)\right) v_{1} \wedge \cdots \wedge v_{d}$, hence $\sigma(e)=\left(\operatorname{det}\left(a_{i j}\right) / \operatorname{det}\left(\sigma\left(a_{i j}\right)\right)\right) e$. But $\operatorname{det}_{\mathbb{Q}_{p}}(V)$ is a one-dimensional representation, so it must be of the form $\phi_{0} \phi \kappa^{k}$, where $\phi_{0}$ is a finite-order $N$ of character, $\phi$ is an unramified character such that $\phi\left(\operatorname{Frob}_{p}\right)=u$ for some $u \in \mathbb{Z}_{p}^{\times}$. Take $\varpi \in \widehat{\mathbb{Q}}_{p}^{u r}$ such that $\operatorname{Frob}_{p}(\varpi)=u \varpi$, then the above identity means that $\operatorname{det}\left(a_{i j}\right) / \sigma\left(\operatorname{det}\left(a_{i j}\right)\right)=\phi_{0}(\sigma) \cdot \phi(\sigma) \cdot \kappa^{k}(\sigma)$. Assume $\sigma \in G_{K_{\infty}}$ and raise $N$ th power, we get $\left(\operatorname{det}\left(a_{i j}\right) / \sigma\left(\operatorname{det}\left(a_{i j}\right)\right)\right)^{N}=\phi^{N}(\sigma)$. Take $\Delta=\left(\operatorname{det}\left(a_{i j}\right) \varpi\right)^{N}$, then this gives $\sigma(\Delta)=\Delta$ for all $\sigma \in G_{K_{\infty}}$. On the other hand, $\varphi(\Delta)=\varphi\left(\operatorname{det}\left(a_{i j}\right) \cdot \varpi\right)^{N}=$ $\operatorname{det}\left(a_{i j}\right)^{N} \cdot \varpi^{N} \cdot u^{N}=u^{N} \cdot \Delta$. Now, taking $\zeta=u^{N}, g: x \rightarrow \Delta x$, then $g\left(e_{i}\right)=\Delta e_{i}$ and $\varphi\left(\Delta e_{i}\right)=u_{N} \delta e_{i}=\zeta \Delta e_{i}$, and the lemma follows.

Corollary 4.5. For $k \in \mathbb{N}$, there exist $\alpha_{k} \in \mathbb{Z}_{p}$ and $g_{k}: t_{\pi}^{-k} B_{\max }^{+} \otimes V \rightarrow\left(B_{\max }^{+}\right)^{d}$ such

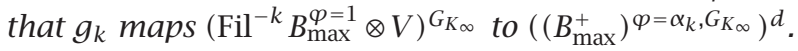


Proof. Use the $g$ in Lemma 4.4 , assume $t_{\pi}^{s} \cdot g\left(B_{\max }^{+} \otimes V\right) \hookrightarrow\left(B_{\max }^{+}\right)^{d}$, and let $g_{k}=$ $t_{\pi}^{k+s} g, \alpha_{k}=\pi^{k+s} \zeta$.

LEMMA 4.6. For $k \in \mathbb{N}, \epsilon>0$, there exists $n \in \mathbb{N}$ such that for $\sigma \in \Gamma_{n}$ and $F \in$ $\left(\mathrm{Fil}^{-k} B_{\max }^{\varphi=1} \otimes V\right)^{G_{K_{\infty}}}$,

$$
\left\|g_{k}(\sigma(F))-\sigma\left(g_{k}(F)\right)\right\| \leq \epsilon\|F\|
$$

Proof. Let $\gamma_{1}$ be a topology generator of $\Gamma_{1}$ and $\tilde{\gamma}_{1} \in G_{\mathbb{Q}_{p}}$ a lifting of $\gamma_{1}$; we can choose $\tilde{\gamma}_{1}$ in the $p$-Sylow subgroup of $G_{\mathbb{Q}_{p}} \cdot \tilde{\gamma}_{1}$ induces a continuous map from $\Gamma_{1} \rightarrow G_{\mathbb{Q}_{p}}$ by sending $x$ to $\widetilde{\gamma_{1}} \log _{\gamma_{1}} x$. So, for $\sigma \in \Gamma_{1}$, we can get $\tilde{\sigma} \in G_{\mathbb{Q}_{p}}$ under such a map. The map $g_{k} \circ \tilde{\sigma}-\tilde{\sigma} \circ g_{k}: B_{\max } \otimes V \rightarrow\left(B_{\max }\right)^{d}$ and the map $g_{k} \circ \sigma-\sigma \circ g_{k}:\left(F^{-k}\left(B_{\max }^{\varphi=1} \otimes V\right)\right)^{G_{K_{\infty}} \rightarrow}$ $\left(B_{\max }^{d}\right)$ are the same if we restrict to $\left(B_{\max }^{\varphi=1} \otimes V\right)^{G_{K_{\infty}}}$, and

$$
g_{k}\left(\tilde{\sigma}\left(t_{\pi}^{-k} v_{i}\right)\right)-\tilde{\sigma} g_{k}\left(t_{\pi}^{-k} v_{i}\right)=g_{k}\left((\tilde{\sigma}-1)\left(t_{\pi}^{-k} v_{i}\right)\right)+(1-\tilde{\sigma}) g_{k}\left(t_{\pi}^{-k} v_{i}\right)
$$

since $t_{\pi}^{-k} v_{1}, \ldots, t_{\pi}^{-k} v_{d}$ are a base of $t_{\pi}^{-k} B_{\max }^{+} \otimes V$ over $B_{\max }^{+}$, and when $\sigma \rightarrow 1$ the above goes to zero, hence the lemma follows.

LEMmA 4.7 (Coleman-Colmez exact sequence). For $\alpha \in \mathbb{Z}_{p}, \alpha \neq 0, r=v_{p}(\alpha)$,

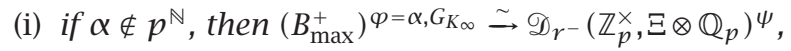

(ii) if $\alpha=p^{r}$, then the following sequence is exact:

$$
0 \longrightarrow \mathbb{Q}_{p} t^{r} \longrightarrow\left(B_{\max }^{+}\right)^{\varphi=p^{r}, G_{K \infty}} \longrightarrow \mathscr{D}_{r^{-}}\left(\mathbb{Z}_{p}^{\times}, \Xi \otimes \mathbb{Q}_{p}\right)^{\psi} \longrightarrow \mathbb{Q}_{p} \longrightarrow 0
$$

Proof. See [2, Appendix A].

LEMMA 4.8. For $r \in \mathbb{R}^{+}$, there exists $C_{r}>0$ such that for $\mu \in \mathscr{D}_{r}\left(\mathbb{Z}_{p}, \Xi \otimes D(V)\right)^{\psi}$ and $a \in \mathbb{Z}_{p}$,

$$
\left\|\sum_{i=0}^{p-1} \delta_{i a} * \mu\right\| \geq C_{r}\|\mu\| .
$$

Proof. This is the same as [2, Lemma III.2.6]. Note the $*$ above is induced by the addition in $\mathbb{Z}_{p}$.

COROLLARY 4.9. For $r \in \mathbb{R}^{+}$, there exists $C_{r}>0$ such that for $\mu \in \mathscr{D}_{r}\left(\mathbb{Z}_{p}, \Xi \otimes D(V)\right)^{\psi}$ and $a \in 1+p \mathbb{Z}_{p}$,

$$
\left\|\sum_{i=0}^{p-1} \delta_{a^{i}} * \mu\right\| \geq C_{r}\|\mu\| .
$$

Here the $*$ is induced by the multiplication in $\mathbb{Z}_{p}^{\times}$.

COROLLARY 4.10. There exist $C>0$ and an $n_{0} \in \mathbb{N}$ such that for $F \in\left(B_{\max }^{+}\right)^{\varphi=\alpha, G_{K_{\infty}}}$ and $\sigma \in \Gamma_{n_{0}}$,

$$
\left\|T_{\sigma}(F)\right\| \geq C\|F\|
$$


Proof. Using Corollary 4.9, $F$ corresponds to $\mu,\|F\|=\|\mu\|$, and $T_{\sigma}(F)$ corresponds to $\sum \delta_{a^{i}} * \mu$, hence

$$
\left\|T_{\sigma}(F)\right\|=\left\|\sum \delta_{a^{i}} * \mu\right\| \geq C\|\mu\|=C\|F\|
$$

COROLlARY 4.11. There exist $a C>0$ and an $n \in \mathbb{N}$ such that $\left\|T_{\sigma}\left(g_{k}(F)\right)\right\| \geq C\left\|g_{k}(F)\right\|$ for all $F \in\left(\mathrm{Fil}^{-k} B_{\max }^{\varphi=1} \otimes V\right)^{G_{K_{\infty}}}$.

Proof. The corollary follows since $g_{k}(F) \in\left(B_{\max }^{+}\right)^{\varphi=\alpha, G_{K \infty}}$.

Now Proposition 4.3 follows since $g_{k}$ is just a multiple by $p^{k+s} \Delta$.

CASE 2 (general $h$ ). In this case we need to show that

$$
p^{n}\left(\sum_{i=0}^{h-1}(-1)^{i}\left(\begin{array}{c}
h-1 \\
i
\end{array}\right)\left(\kappa\left(\sigma_{n}\right)^{-i} \sigma_{n}-1\right) u_{n, i}\right) \rightarrow 0
$$

as $n \rightarrow \infty$.

LEMMA 4.12. For $l \in \mathbb{N}$ and $\sigma \in \Gamma$, put $T_{l, \sigma}=\sum_{j=0}^{p-1} \kappa(\sigma)^{-l j} \sigma^{j}$, then the sequence

$$
p^{n} \prod_{l=0}^{k} T_{l, \sigma_{n}}\left(\sum_{i=0}^{h-1}\left(\begin{array}{c}
h-1 \\
i
\end{array}\right)\left(\kappa\left(\sigma_{n}\right)^{-i} \sigma_{n}-1\right) u_{n, i}\right)
$$

tends to zero as $n \rightarrow \infty$.

Proof. For $l \geq 0$ and $l \leq k$

$$
T_{l, \sigma_{n}}\left(\kappa\left(\sigma_{n}\right)^{-i} \sigma_{n}-1\right)\left(u_{n, i}\right)=\left(\kappa\left(\sigma_{n}^{p}\right)^{-i} \cdot \sigma_{n}^{p}-1\right) u_{n, i}=-\int_{1+p^{n} \mathbb{Z}_{p}} x^{-i} \mu_{\sigma_{n}^{p}},
$$

then (4.25) equals

$$
-p^{n} \sum_{i=0}^{h-1}(-1) i\left(\begin{array}{c}
h-1 \\
i
\end{array}\right) \prod_{l=0, l \neq i}^{h-1} T_{l, \sigma_{n}} \int_{1+p^{n} \mathbb{Z}_{p}} x^{-i} \mu_{\sigma_{n}^{p}}=-p^{n} \sum_{l=0}^{h-1} R_{l} \cdot Y_{l}
$$

with $R_{l} \in p^{n(h-1-l)} \mathbb{Z}_{p}[[T]]$ and $p^{n} R_{l} \cdot Y_{l} \in p^{n(h-l)} \mathbb{Z}_{p}[[T]] \int_{1+p^{n} \mathbb{Z}_{p}}(x-1)^{l} \mu_{\sigma_{n}^{p}} \rightarrow 0$. By [2, Lemma III.3.3], the proof of the lemma follows.

LEMMA 4.13. There exist $a C>0$ and an $n \in \mathbb{N}$ such that

$$
\left\|\prod_{i=0}^{h-1} T_{i, \sigma}(F)\right\| \geq C\|F\|^{h}
$$

for all $F \in\left(\mathrm{Fil}^{-k} B_{\max }^{\varphi=1} \otimes V\right)^{G_{K \infty}}$ and $\sigma \in \Gamma_{n}$. 
Proof. The lemma follows using Proposition $4.3 h$ times.

This completes the proof of Lemma 4.2.

So, this completes the proof of the existence of the logarithm.

COROLlaRY 4.14. For $\mu \in H^{1}\left(K, \mathscr{D}_{h^{-}}\left(\mathbb{Z}_{p}^{\times}, V\right)\right)^{G_{K \infty}}$, if $\mu_{\tau}=0$ for all $\tau \in G_{K_{\infty}}$ and $\int_{1+p^{n} \mathbb{Z}_{p}} x^{-i} \mu \in H_{e}^{1}\left(K_{n}, V(\kappa-i)\right)$, then $\mathbb{L}_{h, V}(\mu) \in\left(B_{\max }^{\varphi=1} \otimes V\right)^{G_{K \infty}}$.

Proof. Since $\left(1-\kappa(\tau)^{-i} \tau\right) c_{n, i}=\int_{1+p^{n} \mathbb{Z}_{p}} x^{-i} \mu_{\tau}$ for $\tau \in G_{K_{\infty}}, \tau\left(c_{n, i}\right)=c_{n, i}$ and $c_{n, i}=$ 0 for $n \gg 1$, hence $\mathbb{L}_{h, V}(\mu) \in\left(B_{\max }^{\varphi=1} \otimes V\right)^{G_{K_{\infty}}}$.

5. The relationship between the exponential map and the logarithmic map. For a crystalline representation $V$, assume $h \in \mathbb{Z}$ such that $\mathrm{Fil}^{-h} D(V)=D(V)$.

Lemma 5.1. For $\mu \in \widetilde{\mathscr{D}}_{\text {temp }}\left(\mathbb{Q}_{p}, \Xi \otimes D(V)\right)^{\Phi=1, \psi}$, there exists $i \gg 1$ such that $\mathscr{F}_{\text {cont }}(\mu /$ $\left.(-t x)^{i}\right)$ exists.

Proof. See [2, Section II.2.3].

Let $\Sigma=\left(\cup_{n=0}^{+\infty}\left(\left(\oplus_{i \in \mathbb{Z}} t^{-i} B_{\max }^{\varphi=1}\right) \otimes V(-i)\right)^{G_{K}}\right)$.

THEOREM 5.2. For $\mu \in \widetilde{\mathscr{D}}_{\text {temp }}\left(\mathbb{Q}_{p}, \Xi \otimes D(V)\right)^{\Phi=1, \psi}$, choose $r$ as in Lemma 2.1 and assume $h \geq 1$ such that $\mathrm{Fil}^{-h} D(V)=D(V)$, then

$$
\mathbb{L}_{(h+r), V(r)}\left(\mathbb{E}_{(h+r), V(r)}\left(\frac{\mu}{(-t x)^{r}}\right)\right) \equiv \int_{\mathbb{Q}_{p}}\left[\varepsilon^{x}\right] \frac{\mu}{(-t x)^{r}} \bmod \Sigma .
$$

Proof. Let $F=\int_{\mathbb{Q}_{p}}\left[\varepsilon^{x}\right]\left(\mu /(-t x)^{r}\right)$, then it is easy to see that $F \in F_{V}^{0}\left(B_{\max }^{\varphi=1} \otimes V(r)\right)^{G_{K_{\infty}}}$. By the definition of $\mathbb{L}_{h, V}$, we only need to calculate, for $0 \leq i \leq h+r-1$, the integral

$$
\begin{aligned}
\int_{1+p^{n} \mathbb{Z}_{p}} x^{-i} \cdot x^{r} \mathbb{E}_{h, V}(\mu) & =\int_{1+p^{n} \mathbb{Z}_{p}} x^{r-i} \mathbb{E}_{h, V}(\mu) \\
& =\exp _{V\left(\kappa^{r-i}\right)}\left(\int_{1+p^{n} \mathbb{Z}_{p}} x^{\left.r-i \mathscr{F}_{\mathrm{alg}}^{(h)}(\mu)\right)}\right. \\
& =\exp _{V\left(\kappa^{r-i}\right)}\left(\int_{\mathbb{Q}_{p}} \mathscr{F}_{\mathrm{alg}}^{(h)}\left(1_{1+p^{n} \mathbb{Z}_{p}} \cdot x^{r-i}\right) \mu\right) \\
& =\exp _{V\left(\kappa^{r-i}\right)}\left(\int_{\mathbb{Q}_{p}} p^{-n} \cdot 1_{p^{-n} \mathbb{Z}_{p}} \cdot \frac{(r-i+h-1) !}{(-t x)^{r-i}} \varepsilon(x) \mu\right) \\
& =\exp _{V\left(\kappa^{r-i}\right)}\left((h+i-1-r) ! i ! \delta_{V\left(\kappa^{r-i}\right)} \circ T_{n}\left(\mathscr{F}_{\operatorname{cont}}\left(\frac{\mu}{(-t x)^{r}}\right)\right)\right),
\end{aligned}
$$

where we have used Proposition 2.7 to get $\delta_{V\left(\kappa^{r-i}\right)} \circ T_{n}\left(\mathscr{F}_{\mathrm{cont}}\left(\mu /(-t x)^{r}\right)\right)$. Let $d_{n, i}=$ $(-1)^{i}(h+r-1-i) ! i ! \delta_{V\left(\kappa^{i-r}\right)} \circ T_{n}\left(\mathscr{F}_{\text {cont }}\left(\mu /(-t x)^{r}\right)\right)$ and $c_{n, i}=e_{B} \circ$ Fil $^{<0}\left(d_{n, i}\right)$. Then $c_{n, i} \in$ $B_{\max }^{\varphi=1} \otimes V$ such that the above cohomology class is represented by the cocycle

$$
\boldsymbol{T} \longrightarrow(1-\tau) \circ c_{n, i}=\left(1-\kappa^{r-i} \boldsymbol{T}\right) c_{n, i}
$$


By Theorem 4.1, $\mathbb{E}_{h+r, V\left(\kappa^{r}\right)}\left(\mu /(-t x)^{r}\right)$ is tempered in $H^{1}\left(K_{\infty}, \mathscr{D}_{(h+r+r(V))^{-}}\left(\mathbb{Z}_{p}^{\times}, V\right)\right)^{\Gamma}$, under the inflation-restriction exact sequence

$$
\begin{aligned}
0 & \longrightarrow H^{1}\left(\Gamma, \mathscr{D}_{(h+r+r(V))^{-}}\left(\mathbb{Z}_{p}^{\times}, V\right)^{G_{K_{\infty}}}\right) \longrightarrow H^{1}\left(\mathbb{Q}_{p}, \mathscr{D}_{(h+r+r(V))^{-}}\left(\mathbb{Z}_{p}^{\times}, V\right)\right) \\
& \longrightarrow H^{1}\left(K_{\infty}, \mathscr{D}_{(h+r+r(V))^{-}}\left(\mathbb{Z}_{p}^{\times}, V\right)\right)^{\Gamma} \longrightarrow 0 ;
\end{aligned}
$$

we can lift $\mathbb{E}_{h+r, V\left(\kappa^{r}\right)}\left(\mu /(-t x)^{r}\right)$ to an element in $H^{1}\left(\mathbb{Q}_{p}, \mathscr{D}_{(h+r+r(V))^{-}}\left(\mathbb{Z}_{p}^{\times}, V\right)\right)$, the lifting is not unique. By Corollary 4.14, modulo $\left(B_{\max }^{\varphi=1} \otimes V\right)^{G_{K_{\infty}}}$ the left-hand side of (5.1) is equal to

$$
\begin{aligned}
& \frac{1}{(h+r-1) !} \cdot p^{n} \sum_{i=0}^{h+r-1}(-1)^{i} \cdot\left(\begin{array}{c}
h+r-1 \\
i
\end{array}\right) \cdot c_{n, i} \\
& =\frac{1}{(h+r-1) !} p^{n} \cdot \sum_{i=0}^{h+r-1}(-1)^{i}\left(\begin{array}{c}
h+r-1 \\
i
\end{array}\right) \\
& \cdot e_{B} \circ \mathrm{Fil}^{<0}\left((-1)^{i}(h+r-1-i) ! i ! \delta_{V\left(\kappa^{i-r}\right)} T_{n}\left(\mathscr{F}_{\operatorname{cont}}\left(\frac{\mu}{(-t x)^{r}}\right)\right)\right) \\
& =p^{n} \sum_{i=0}^{h+r+1} e_{B} \circ \text { Fil }^{<0} \circ \delta_{V\left(\kappa^{i-r}\right)}\left(T_{n}(F)\right) \\
& =p^{n} e_{B} \circ \text { Fil }^{<0} \sum_{i=0}^{h+r-1} \delta_{V\left(\kappa^{i-r}\right)} T_{n}(F) \\
& =p^{n} e_{B} \circ \mathrm{Fil}^{<0}\left(T_{n}(F)\right) \\
& =e_{B} \circ \operatorname{Fil}^{<0}\left(p^{n} T_{n}(F)\right) \\
& \longrightarrow e_{B} \circ \mathrm{Fil}^{<0}(F)=\mathrm{Fil}^{<0}(F)=F .
\end{aligned}
$$

THEOREM 5.3. Assume $V$ is a de Rham representation, $h \geq 1$ an integer, and $\mu \in$ $H^{1}\left(\mathbb{Q}_{p}, \mathscr{D}_{h^{-}}\left(\mathbb{Z}_{p}^{\times}, V\right)\right)$ such that $\int_{1+p^{n} \mathbb{Z}_{p}} x^{-i} \mu \in H_{e}^{1}\left(K_{n}, V\left(\kappa^{-i}\right)\right)$ for $n \geq 1$ and $0 \leq i \leq h-1$, then

$$
\delta_{V\left(\kappa^{-k}\right)} \circ T_{m}\left(\mathbb{L}_{h, V}(\mu)\right)=\exp _{V\left(\kappa^{k}\right)}^{*}\left(\frac{(-1)^{h-1}}{k(k-1) \cdots(k-h+1)} \int_{1+p^{m} \mathbb{Z}_{p}} x^{-k} \mu\right) .
$$

Proof. We will use the isomorphism $\kappa: \Gamma \rightarrow \mathbb{Z}_{p}^{\times}, \Gamma_{n} \mapsto 1+p^{n} \mathbb{Z}_{p}$, to transfer the measure on $\mathbb{Z}_{p}^{\times}$to the measures on $\Gamma$. Assume $\gamma_{0}$ is a topology generator of $\Gamma$ and $\gamma_{n}=\gamma_{0}^{\left[K_{n}: \mathbb{Q}_{p}\right]}$ is a generator of $\Gamma_{n}$. It follows that

$$
\delta_{V\left(\kappa^{-i}\right)} \circ T_{m}\left(p^{n} u_{n, i}\right)=t_{\pi}^{i} \delta_{V\left(\kappa^{i-k}\right)} \circ T_{m}\left(p^{n} t_{\pi}^{-i} u_{n, i}\right),
$$

for $\gamma \in \Gamma_{m}$,

$$
\delta_{V\left(\kappa^{i-k}\right)} \circ T_{m} \circ \gamma=\kappa(\gamma)^{k-i} \circ T_{m},
$$


hence

$$
\begin{gathered}
\frac{1-\kappa\left(\gamma_{n}\right)^{k-i}}{p^{n}} \delta_{V\left(\kappa^{i-k}\right)} \circ T_{m}\left(p^{n} t_{\pi}^{-i} u_{n, i}\right)=\delta_{V\left(\kappa^{i-k}\right)} \circ T_{m}\left(\left(1-\gamma_{n}\right) t_{\pi}^{-i} u_{n, i}\right), \\
\left(1-\gamma_{n}\right) t_{\pi}^{-i} u_{n, i}=t_{\pi}^{-i} \int_{\Gamma_{n}} \kappa(x)^{-i} \mu_{\gamma_{n}} .
\end{gathered}
$$

The cocycle relation gives

$$
\mu_{\gamma_{n}}=\sum_{l=0}^{\left[K_{n}: K_{m}\right]-1} t_{\pi}^{-i} \gamma_{m}^{l}\left(\int_{\gamma_{m}^{-l} \Gamma_{m}} \kappa\left(\gamma_{m}^{l} x\right)^{-i} \mu_{\gamma_{m}}\right)
$$

and we get

$$
\begin{aligned}
\delta_{V\left(\kappa^{i-k}\right)} & \circ T_{m}\left(\int_{\Gamma_{n}}\left(t_{\pi} \kappa(x)\right)^{-i} \mu_{\gamma_{n}}\right) \\
= & t_{\pi}^{-i} \delta_{V\left(\kappa^{-k}\right)} \circ T_{m}\left(\sum_{l=0}^{\left[K_{n}: K_{m}\right]-1} \kappa(\gamma)^{l(k-i)} \int_{\gamma_{m}^{-1} \Gamma} \kappa(x)^{-i} \mu_{\gamma_{m}}\right) .
\end{aligned}
$$

The element $\delta_{V\left(\kappa^{-k}\right)} \circ T_{m}\left(\mathbb{L}_{h, V}(\mu)\right)$ is the limit of

$$
\frac{p^{n}}{1-\kappa\left(\gamma_{n}\right)^{k-i}} \delta_{V\left(\kappa^{-k}\right)} \circ T_{m}\left(\sum_{i=0}^{h-1}\left(\begin{array}{c}
h-1 \\
i
\end{array}\right)(-1)^{i} \cdot \sum_{l=0}^{\left[K_{n}: K_{m}\right]-1} \kappa\left(\gamma_{m}\right)^{l(k-i)} \int_{\gamma_{m}^{-l} \Gamma_{n}} \kappa(x)^{-i} \mu_{\gamma_{m}}\right) \text {. }
$$

CASE $1(h=1)$. The above formula becomes

$$
\begin{gathered}
\frac{p^{n}}{1-\kappa\left(\gamma_{n}\right)^{k}} \cdot \delta_{V\left(\kappa^{-k}\right)} \circ T_{m}\left(\sum_{l=0}^{\left[K_{n}: K_{m}\right]-1} \kappa\left(\gamma_{m}\right)^{l(k-i)} \int_{\gamma_{m}^{-l} \Gamma_{n}} \kappa(x)^{-i} \mu_{\gamma_{m}}\right) \\
=\frac{p^{n}}{1-\kappa\left(\gamma_{n}\right)^{k}} \cdot \delta_{V\left(\kappa^{-k}\right)} \circ T_{m}\left(\sum_{l=0}^{\left[K_{n}: K_{m}\right]-1} \kappa\left(\gamma_{m}\right)^{l k} \int_{\gamma_{m}^{-l} \Gamma_{n}} \mu_{\gamma_{m}}\right) \\
\longrightarrow\left\{\begin{array}{l}
\frac{p^{m}}{k \log \kappa\left(\gamma_{m}\right)} \delta_{V\left(\kappa^{-k}\right)} \circ T_{m}\left(\int_{\Gamma_{m}} \kappa(x)^{-k} \mu_{\gamma_{m}}\right), \quad \text { if } m \geq 1, \\
\frac{p}{p-1} \cdot \frac{1}{k \log \kappa\left(\gamma_{0}\right)} \cdot \delta_{V\left(\kappa^{-k}\right)} \circ T_{0}\left(\int_{\Gamma_{0}} \kappa(x)^{-k} \mu_{\gamma_{0}}\right) .
\end{array}\right.
\end{gathered}
$$

Hence, we come to the formula

$$
\delta_{V\left(\kappa^{-k}\right)} \circ T_{m}\left(\mathbb{L}_{1, V}(\mu)\right)=\frac{1}{k \log \kappa\left(\gamma_{m}\right)} \delta_{V\left(\kappa^{-k}\right)} \circ \operatorname{Tr}_{/ K_{m}}\left(\int_{\Gamma_{m}} \kappa(x)^{-k} \mu_{\gamma_{m}}\right) .
$$

Therefore, the theorem follows from the following lemma.

LEMMA 5.4 (Kato [5]). If $V$ is a de Rham representation of $G_{K}$, for any unramified character $\psi$, the map which sends $x \in D(V)$ to the cocycle $\tau \rightarrow x \log \psi(\tau) \in D(V) \subset$ $B_{d R} \otimes V$ is the zero map, and the map which sends $x$ to $\tau \rightarrow x \log \chi(\tau)$ (hence $\tau \rightarrow$ $x \log \kappa(\tau))$ gives an isomorphism of $D(V)$ to $H^{1}\left(K, B_{d R} \otimes V\right)$, and $\exp _{V}^{*}$ coincides with the 
map $H^{1}(K, V) \rightarrow H^{1}\left(K, B_{d R} \otimes V\right) \rightarrow D(V)$, where the last map is the inverse map of the above isomorphism.

For any $K \subset K_{\infty}$ and a de Rham representation $V$ of $G_{K}$, we have the following diagram:

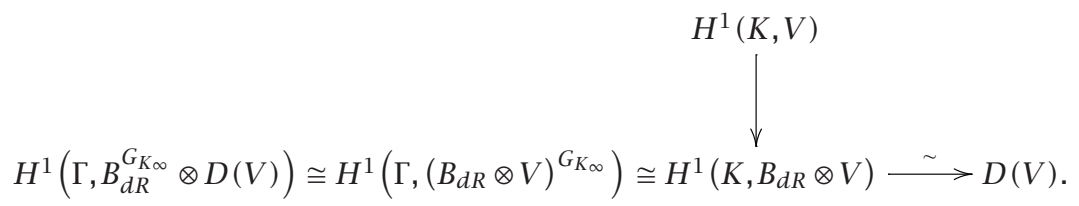

If $c \in H^{1}(K, V)$ and $\tau \rightarrow c_{\tau}$ is the cocycle representation, we can choose $c^{\prime} \in H^{1}\left(\Gamma, B_{d R}^{G_{K \infty}} \otimes\right.$ $D(V))$ such that $c$ and $c^{\prime}$ have the same images in $H^{1}\left(K, B_{d R} \otimes V\right)$. Let $\tau \rightarrow c_{\tau}^{\prime}$ be a cocycle representation of $c^{\prime}$. The element $\delta_{V} \circ \operatorname{Tr}_{/ K}\left(c_{\gamma}^{\prime} / \log _{p} \kappa(\gamma)\right) \in D(V)$. Using Kato's lemma, we see that it is the same as $\exp _{V}^{*}(c)$, hence

$$
\exp _{V}^{*}(c)=\delta_{V} \circ \operatorname{Tr}_{/ K}\left(\frac{1}{\log \kappa(\gamma)} c_{\gamma}\right)
$$

Using this formula for $K=K_{m}, V=V\left(\kappa^{-k}\right)$ (hence $\left.\gamma=\gamma_{m}\right), c=\int_{1+p^{m} \mathbb{Z}_{p}} x^{-k} \mu \in H^{1}\left(K_{m}\right.$, $\left.V\left(\kappa^{-k}\right)\right)$, then

$$
\exp _{V\left(\kappa^{-k}\right)}^{*}\left(\int_{1+p^{m} \mathbb{Z}_{p}} x^{-k} \mu\right)=\delta_{V\left(\kappa^{-k}\right)} \circ \operatorname{Tr}_{/ K_{m}}\left(\frac{1}{\log \kappa(\gamma)} \int_{\Gamma_{m}} \kappa^{-k}(x) \mu_{\gamma_{m}}\right) .
$$

This completes the proof of the theorem for Case 1; the general case follows an argument similar to [2, Section 3.3].

6. Explicit reciprocity law. By constructing the logarithmic map, now we can evaluate the integral of the analytic cohomology class at "negative" power $(\leq-h)$.

LEMmA 6.1. Assume $V$ is a crystalline representation and $h \in \mathbb{Z}, h \geq 1$, such that $\mathrm{Fil}^{-h} D(V)=D(V), F \in \Sigma=\oplus_{i \in \mathbb{Z}} \cup_{n=0}^{+\infty} F_{V}^{(0)}\left(B_{\max }^{\varphi=1} \otimes V\left(\kappa^{-i}\right)\right)^{G_{K n}}$, then $\delta_{V\left(\kappa^{-k}\right)} \circ T_{m}(F)=0$ for $k \geq h$.

Proof. We can assume $F \in F_{V}^{0}\left(B_{\max }^{\varphi=1} \otimes V(-i)\right)^{G_{K} n_{0}}$ for some $n_{0} \gg 1$ and some $i$. Since $\Sigma$ is a finite-dimensional $\mathbb{Q}_{p}$-vector space, $F \in B_{d R}^{0} \otimes D(V)$, hence $F=\sum b_{i} \otimes d_{i}$.

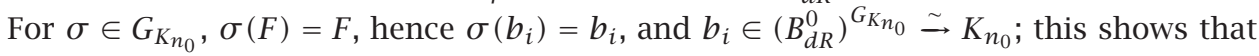
$T_{m}(F) \in D(V)$. Hence, $\delta_{V\left(\kappa^{-k}\right)} \circ T_{m}(F)=0$ for $k \geq 1$. This proves the lemma.

THEOREM 6.2. Assume $V$ is a crystalline representation of $G_{\mathbb{Q}_{p}}$ and suppose $h \in \mathbb{Z}$ and $\mu \in \tilde{\mathscr{D}}_{\text {temp }}\left(\mathbb{Q}_{p}, \Xi \otimes D(V)\right)^{\Phi=1, \psi}, k \geq h$, then

$$
\begin{gathered}
\exp _{V\left(\kappa^{-k}\right)}^{*}\left(\int_{\mathbb{Z}_{p}^{\times}} x^{-k} \mathbb{E}_{h, V}(\mu)\right)=(-1)^{h-1} \cdot(1-\varphi)^{-1}\left(1-p^{-1} \varphi^{-1}\right)\left(\int_{\mathbb{Z}_{p}^{\times}} \frac{(t x)^{k}}{(k-h) !} \mu\right), \\
\exp _{V\left(\kappa^{-k}\right)}^{*}\left(\int_{a+p^{n} \mathbb{Z}_{p}} x^{-k} \mathbb{E}_{h, V}(\mu)\right)=(-1)^{h-1} \frac{\varphi^{-n}}{p^{n}}\left(\int_{\mathbb{Z}_{p}} \varepsilon\left(\frac{a x}{p^{n}}\right) \frac{(t x)^{k}}{(k-h) !} \mu\right) .
\end{gathered}
$$


Proof. First of all, assume $h \geq 1$ and $\mathrm{Fil}^{-h} D(V)=D(V)$. Assume $r \in \mathbb{N}$ is sufficiently large such that $F_{r}=\mathscr{F}_{\text {cont }}\left(\mu /(-t x)^{r}\right)$ exists. Using Theorem $5.3, h+r$ for $h$, $V\left(\kappa^{r}\right)$ for $V$, and $\mathbb{E}_{h+r, V\left(\kappa^{r}\right)}\left(\mu /(-t x)^{r}\right)$ for $\mu$, we get

$$
\begin{aligned}
& \delta_{V\left(\kappa^{-k}\right)} \circ T_{m}\left(\mathbb{L}_{h+r, V(r)} \circ \mathbb{E}_{h+r, V(r)}\left(\frac{\mu}{(-t x)^{r}}\right)\right) \\
& =\exp _{V\left(\kappa^{k}\right)}^{*}\left(\frac{(-1)^{h+r-1}(k-h) !}{(k+r) !}\left(\int_{1+p^{m} \mathbb{Z}_{p}} x^{-k-r} \cdot x^{r} \mathbb{E}_{h, V}(\mu)\right)\right) .
\end{aligned}
$$

By Theorem 5.2 and Lemma 6.1, this gives

$$
\exp _{V\left(\kappa^{k}\right)}^{*}\left(\int_{1+p^{m} \mathbb{Z}_{p}} x^{-k} \mathbb{E}_{h, V}(\mu)\right)=(-1)^{h+r-1} \frac{(k+r) !}{(k-h) !} \delta_{V\left(\kappa^{-k}\right)} \circ T_{m}\left(\mathscr{F}_{\operatorname{cont}}\left(\frac{\mu}{(-t x)^{r}}\right)\right) .
$$

CASE $1(m=0)$. By Proposition 2.7, it gives

$$
\begin{gathered}
(-1)^{h+r-1} \frac{(k+r) !}{(k-h) !} \cdot(-1)^{r} \cdot(1-\varphi)^{-1}\left(1-p^{-1} \varphi^{-1}\right) \int_{\mathbb{Z}_{p}^{\times}} \frac{(t x)^{k}}{(k+r) !} \mu \\
=(-1)^{h-1}(1-\varphi)^{-1}\left(1-p^{-1} \varphi^{-1}\right) \int_{\mathbb{Z}_{p}^{\times}} \frac{(t x)^{k}}{(k-h) !} \mu .
\end{gathered}
$$

CASE $2(m=n \geq 1)$. By Proposition 2.7, the above formula gives

$$
(-1)^{h+r-1} \frac{(k+r) !}{(k-h) !} \cdot p^{-n} \cdot \int_{p^{-n} \mathbb{Z}_{p}} \varepsilon(x) \frac{(t x)^{k+r}}{(k+r) !} \frac{\mu}{(-t x)^{r}}=(-1)^{h-1} \int_{\mathbb{Z}_{p}} \varepsilon\left(\frac{x}{p^{n}}\right) \frac{(t x)^{k}}{(k-h) !} \mu .
$$

This completes the proof of the theorem for this special case of $h$, the case for general $h$ follows from the relation $\mathbb{E}_{h, V}=l_{h-1} \mathbb{E}_{h-1, V}$, where $l_{h-1}=h \delta_{1}+\delta_{1}^{\prime}$.

Define the pairing

$$
[\cdot, \cdot]_{D(V)}: \widetilde{\mathscr{D}}_{\mathrm{temp}}\left(\mathbb{Q}_{p}, \Xi \otimes D(V)\right)^{\Phi=1, \psi} \times \widetilde{\mathscr{D}}_{\mathrm{temp}}\left(\mathbb{Q}_{p}, \Xi \otimes D\left(V^{*}(1)\right)\right)^{\Phi=1, \psi} \longrightarrow \mathscr{D}_{\mathrm{temp}}\left(\mathbb{Z}_{p}^{\times}, \mathbb{Q}_{p}\right)
$$

by the formula

$$
\int_{\mathbb{Z}_{p}^{\times}} f(x)\left[\mu, \mu^{\prime}\right]_{D(V)}:=\int_{\mathbb{Z}_{p}^{\times} \times \mathbb{Z}_{p}^{\times}} f\left(x^{-1} y\right) \mu \otimes \mu^{\prime} .
$$


We must check that the integral really takes values in $\mathbb{Q}_{p}$. From the definition of $\mu$ and $\mu^{\prime}$, we know that $\left[\mu, \mu^{\prime}\right]$ will take values in $\Xi$. For any Galois element $\sigma$, we have

$$
\begin{aligned}
\sigma\left(\int_{\mathbb{Z}_{p}^{\times} \times \mathbb{Z}_{p}^{\times}} f\left(x^{-1} y\right) \mu \otimes \mu^{\prime}\right) & =\int_{\mathbb{Z}_{p}^{\times} \times \mathbb{Z}_{p}^{\times}} f\left((\psi(\sigma) x)^{-1}(\psi(\sigma) y)\right) \mu \otimes \mu^{\prime} \\
& =\int_{\mathbb{Z}_{p}^{\times} \times \mathbb{Z}_{p}^{\times}} f\left(x^{-1} y\right) \mu \otimes \mu^{\prime}
\end{aligned}
$$

so $\left[\mu, \mu^{\prime}\right]$ takes values in $\mathbb{Q}_{p}$, and the above pairing is well defined.

Define the paring $\left(\mu, \mu^{\prime}\right)_{V}$ to be

$$
\begin{aligned}
H^{1}\left(\mathbb{Q}_{p},\right. & \left.\mathscr{D}_{\text {temp }}\left(\mathbb{Z}_{p}^{\times}, V\right)\right) \times H^{1}\left(\mathbb{Q}_{p}, \mathscr{D}_{\text {temp }}\left(\mathbb{Z}_{p}^{\times}, V^{*}(1)\right)\right) \\
& \longrightarrow H^{2}\left(\mathbb{Q}_{p}, \mathscr{D}_{\text {temp }}\left(\mathbb{Z}_{p}^{\times} \times \mathbb{Z}_{p}^{\times}, \mathbb{Q}_{p}(1)\right)\right) \\
& \longrightarrow \mathscr{D}_{\text {temp }}\left(\mathbb{Z}_{p}^{\times}, H^{2}\left(\mathbb{Q}_{p}, \mathbb{Q}_{p}(1)\right)\right) \\
& \longrightarrow \mathscr{D}_{\text {temp }}\left(\mathbb{Z}_{p}^{\times}, \mathbb{Q}_{p}\right),
\end{aligned}
$$

where for $\xi \in H^{2}\left(K, \mathscr{D}_{\text {temp }}\left(\mathbb{Z}_{p}^{\times} \times \mathbb{Z}_{p}^{\times}, \mathbb{Q}_{p}(1)\right)\right)$, we associate a distribution $\mu$ as

$$
\int_{\mathbb{Z}_{p}^{\times}} f(x) \mu=\int_{\mathbb{Z}_{p}^{\times} \times \mathbb{Z}_{p}^{\times}} f\left(x y^{-1}\right) \xi(x, y) .
$$

We need to check that $\int_{\mathbb{Z}_{p}^{\times}} f(x) \mu$ is a cocycle. This is equivalent to checking that $\int_{\mathbb{Z}_{p}^{\times}} f(x) \sigma \mu=\sigma\left(\int_{\mathbb{Z}_{p}^{\times}} f(x) \mu\right)$ since

$$
\int_{\mathbb{Z}_{p}^{\times}} f(x) \sigma \mu=\sigma\left(\int_{\mathbb{Z}_{p}^{\times} \times \mathbb{Z}_{p}^{\times}} f\left(\kappa(\sigma) x(\kappa(\sigma) y)^{-1}\right) \xi\right)=\sigma\left(\int_{\mathbb{Z}_{p}^{\times}} f(x) \mu\right) .
$$

LEMMA 6.3. (i) For $\mu \in \widetilde{\mathscr{D}}_{\text {temp }}\left(\mathbb{Q}_{p}, \Xi \otimes D(V)\right)^{\Phi=1, \psi}$ and $\mu^{\prime} \in \widetilde{\mathscr{D}}_{\mathrm{temp}}\left(\mathbb{Q}_{p}, \Xi \otimes D\left(V^{*}(1)\right)\right)^{\Phi=1, \psi}$,

$$
\int_{\mathbb{Z}_{p}^{\times}} x^{i}\left[\mu, \mu^{\prime}\right]_{D(V)}=\left[\int_{\mathbb{Z}_{p}^{\times}} x^{-i} \mu, \int_{\mathbb{Z}_{p}^{\times}} x^{i} \mu^{\prime}\right]_{D(V)},
$$

where the last pairing is defined in [8, Section 2].

(ii) For $\xi \in H^{1}\left(\mathbb{Q}_{p}, \mathscr{D}_{\text {temp }}\left(\mathbb{Z}_{p}^{\times}, V\right)\right)$ and $\xi^{\prime} \in H^{1}\left(\mathbb{Q}_{p}, \mathscr{D}_{\text {temp }}\left(\mathbb{Z}_{p}^{\times}, V^{*}(1)\right)\right)$,

$$
\int_{\mathbb{Z}_{p}^{\times}} x^{i}\left(\xi, \xi^{\prime}\right)_{V}=\int_{\mathbb{Z}_{p}^{\times}} x^{i} \xi \cup \int_{\mathbb{Z}_{p}^{\times}} x^{-i} \xi^{\prime}
$$

where the cup product is given by

$$
H^{1}\left(\mathbb{Q}_{p}, V\left(\kappa^{i}\right)\right) \cup H^{1}\left(\mathbb{Q}_{p}, V^{*}\left(\chi \kappa^{-i}\right)\right) \longrightarrow H^{2}\left(\mathbb{Q}_{p}, \mathbb{Q}_{p}(1)\right) \simeq \mathbb{Q}_{p}
$$

(iii) If $\xi$ or $\xi^{\prime}$ restricted to $K_{\infty}$ is zero, then $\left(\xi, \xi^{\prime}\right)=0$.

(iv) The pairing $[\cdot, \cdot]_{D(V)}$ is sesquilinear for the first variable, linear for the second variable, that is, for $\delta \in \mathscr{D}_{0}\left(\mathbb{Z}_{p}^{\times}, \mathbb{Q}_{p}\right)$,

$$
\left[\delta * \mu, \mu^{\prime}\right]_{D(V)}=\delta^{\sqrt{ }} *\left[\mu, \mu^{\prime}\right]_{D(V)}, \quad\left[\mu, \delta * \mu^{\prime}\right]_{D(V)}=\delta *\left[\mu, \mu^{\prime}\right]_{D(V)} .
$$


(v) The pairing $(\cdot, \cdot)_{V}$ is linear for the first variable, sesquilinear for the second variable, that is, for $\delta \in \mathscr{D}_{0}\left(\mathbb{Z}_{p}^{\times}, \mathbb{Q}_{p}\right)$,

$$
\left(\delta * \xi, \xi^{\prime}\right)_{V}=\delta *\left(\xi, \xi^{\prime}\right)_{V}, \quad\left(\xi, \delta * \xi^{\prime}\right)_{V}=\delta^{\sqrt{ }} *\left(\xi, \xi^{\prime}\right)_{V}
$$

Proof. The only thing we need to prove is (iii) and this follows from Lemma 2.10.

Hence, $(\cdot, \cdot)_{V}$ induces a pairing

$$
(\cdot, \cdot)_{V}: H^{1}\left(K_{\infty}, \mathscr{D}_{\text {temp }}\left(\mathbb{Z}_{p}^{\times}, V\right)\right)^{\Gamma} \times H^{1}\left(K_{\infty}, \mathscr{D}_{\text {temp }}\left(\mathbb{Z}_{p}^{\times}, V^{*}(1)\right)\right)^{\Gamma} \longrightarrow \mathscr{D}_{\text {temp }}\left(\mathbb{Z}_{p}^{\times}, \mathbb{Q}_{p}\right) .
$$

THEOREM 6.4. Assume $h \in \mathbb{Z}$ and $V$ is a crystalline representation. For $\mu \in \widetilde{D}_{\text {temp }}\left(\mathbb{Q}_{p}, \Xi\right.$ $\otimes D(V))^{\Phi=1, \psi}$ and $\mu^{\prime} \in \widetilde{\mathscr{D}}_{\text {temp }}\left(\mathbb{Q}_{p}, \Xi \otimes D\left(V^{*}(1)\right)\right)^{\Phi=1, \psi}$,

$$
\left(\mathbb{E}_{h, V}(\mu), \mathbb{E}_{1-h, V^{*}(1)}\left(\mu^{\prime}\right)\right)=(-1)^{h}\left[\delta_{-1} * \mu, \mu^{\prime}\right] .
$$

Proof. For $i \gg 0$, by Theorem 3.3, we have

$$
\int_{\mathbb{Z}_{p}^{\times}} x^{i} \mathbb{E}_{h, v}(\mu)=(h+i-1) ! \exp _{V\left(\kappa^{i}\right)}\left((1-\varphi)^{-1}\left(1-p^{-1} \varphi^{-1}\right) \int_{\mathbb{Z}_{p}^{\times}} \frac{\mu}{(-t x)^{i}}\right)
$$

and by Theorem 6.2, we have

$$
\exp _{V\left(\kappa^{i}\right)}^{*} \int_{\mathbb{Z}_{p}^{\times}} x^{-i} \mathbb{E}_{1-h, V^{*}(1)}\left(\mu^{\prime}\right)=(-1)^{h}(1-\varphi)^{-1}\left(1-p^{-1} \varphi^{-1}\right) \int_{\mathbb{Z}_{p}^{\times}} \frac{(t x)^{i}}{(i-1+h) !} .
$$

Then the theorem follows from the definition of Bloch-Kato dual exponential map and the duality between $1-\varphi$ and $1-p^{-1} \varphi^{-1}$ in [2, Lemma IV.4.6].

\section{REFERENCES}

[1] Y. Amice and J. Vélu, Distributions p-adiques associées aux séries de Hecke, Journées Arithmétiques de Bordeaux (Conf., Univ. Bordeaux, Bordeaux, 1974), Société Mathématique de France, Paris, 1975, pp. 119-131 (French).

[2] P. Colmez, Théorie d'Iwasawa des représentations de de Rham d'un corps local [Iwasawa theory of de Rham representations of a local field], Ann. of Math. (2) 148 (1998), no. 2, 485-571 (French).

[3] E. de Shalit, Iwasawa Theory of Elliptic Curves with Complex Multiplication, Perspectives in Mathematics, vol. 3, Academic Press, Massachusetts, 1987.

[4] M. Hazewinkel, Formal Groups and Applications, Pure and Applied Mathematics, vol. 78, Academic Press, New York, 1978.

[5] K. Kato, Lectures on the approach to Iwasawa theory for Hasse-Weil L-functions via $B_{\mathrm{dR}}$. I, Arithmetic Algebraic Geometry (Trento, 1991), Lecture Notes in Math., vol. 1553, Springer-Verlag, Berlin, 1993, pp. 50-163.

[6] B. Perrin-Riou, Théorie d'Iwasawa des représentations p-adiques sur un corps local, Invent. Math. 115 (1994), no. 1, 81-161 (French).

[7] A. Wiles, Higher explicit reciprocity laws, Ann. of Math. (2) 107 (1978), no. 2, 235-254.

[8] S. Zhang, On a trivial zero problem, Int. J. Math. Math. Sci. 2004 (2004), no. 6, 295-318.

Shaowei Zhang: Department of Mathematics and Statistics, Boston University, MA 02215, USA E-mail address: swz@math.bu.edu 


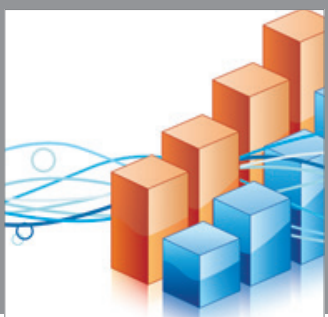

Advances in

Operations Research

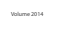

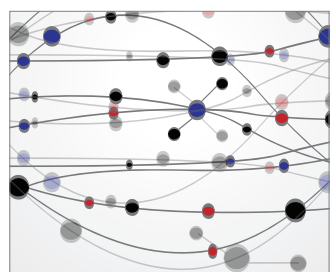

\section{The Scientific} World Journal
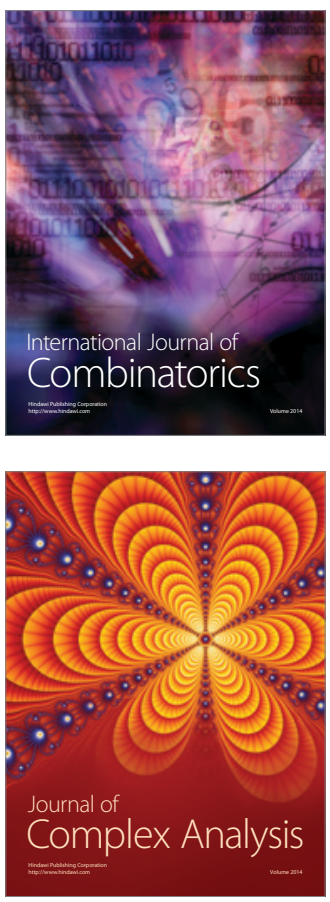

International Journal of

Mathematics and

Mathematical

Sciences
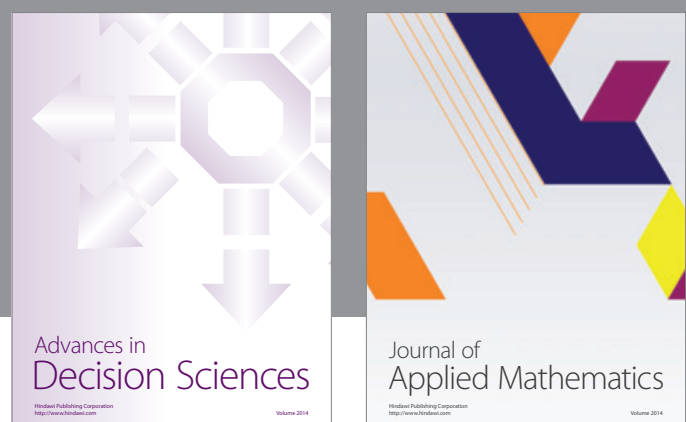

Journal of

Applied Mathematics
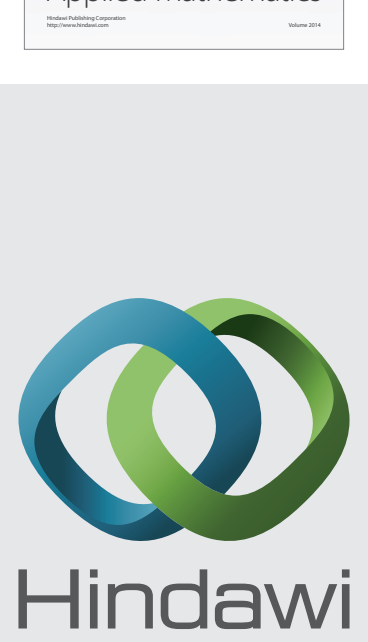

Submit your manuscripts at http://www.hindawi.com
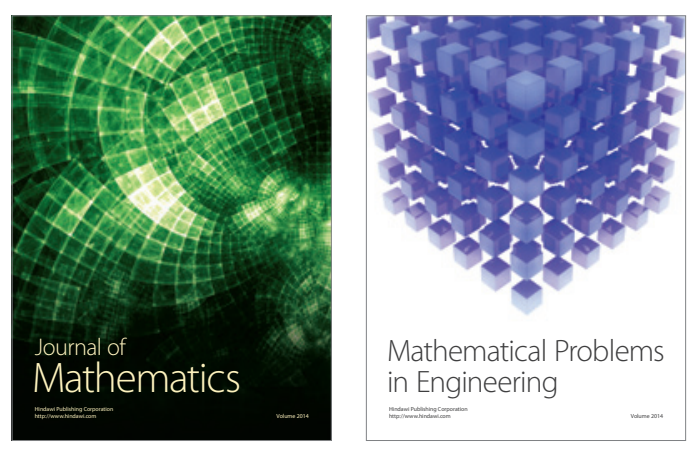

Mathematical Problems in Engineering
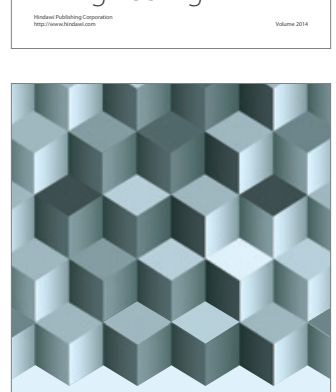

Journal of

Function Spaces
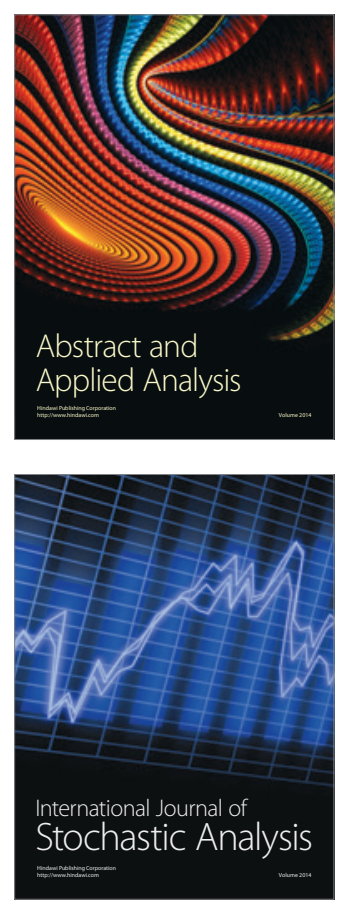

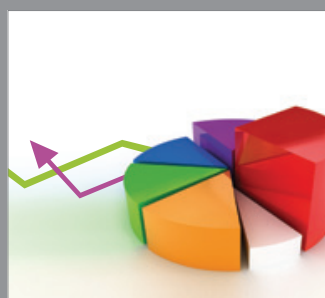

ournal of

Probability and Statistics

Promensencen
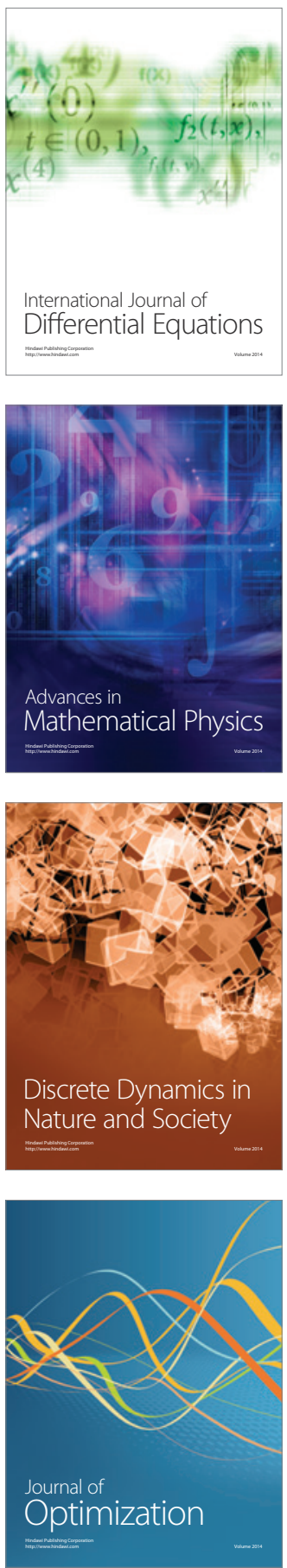